\title{
Investigation of Synapse Formation and Function in a Glutamatergic-GABAergic Two-Neuron Microcircuit
}

\author{
Chia-Ling Chang, ${ }^{1,2,3,4}$ Thorsten Trimbuch, ${ }^{1,3}$ Hsiao-Tuan Chao, ${ }^{5}$ Julia-Christine Jordan, ${ }^{1,3}$ Melissa A. Herman, ${ }^{1,3}$ \\ and Christian Rosenmund ${ }^{1,3,4}$ \\ ${ }^{1}$ Neuroscience Research Center, ${ }^{2}$ Graduate Program in Medical Neuroscience, and ${ }^{3}$ NeuroCure Cluster of Excellence, Charité-Universitätsmedizin Berlin, \\ 10117 Berlin, Germany, and ${ }^{4}$ Departments of Neuroscience and Molecular and Human Genetics and ${ }^{5} J a n$ and Dan Duncan Neurological Research Institute, \\ Department of Pediatrics, Baylor College of Medicine, Houston, Texas 77030
}

\begin{abstract}
Neural circuits are composed of mainly glutamatergic and GABAergic neurons, which communicate through synaptic connections. Many factors instruct the formation and function of these synapses; however, it is difficult to dissect the contribution of intrinsic cell programs from that of extrinsic environmental effects in an intact network. Here, we perform paired recordings from two-neuron microculture preparations of mouse hippocampal glutamatergic and GABAergic neurons to investigate how synaptic input and output of these two principal cells develop. In our reduced preparation, we found that glutamatergic neurons showed no change in synaptic output or input regardless of partner neuron cell type or neuronal activity level. In contrast, we found that glutamatergic input caused the GABAergic neuron to modify its output by way of an increase in synapse formation and a decrease in synaptic release efficiency. These findings are consistent with aspects of GABAergic synapse maturation observed in many brain regions. In addition, changes in GABAergic output are cell wide and not target-cell specific. We also found that glutamatergic neuronal activity determined the AMPA receptor properties of synapses on the partner GABAergic neuron. All modifications of GABAergic input and output required activity of the glutamatergic neuron. Because our system has reduced extrinsic factors, the changes we saw in the GABAergic neuron due to glutamatergic input may reflect initiation of maturation programs that underlie the formation and function of in vivo neural circuits.
\end{abstract}

Key words: GABAergic neuron; release probability; synapse formation; cell autonomous; cell culture; activity dependent

\section{Introduction}

Proper balance of excitation and inhibition (E/I) is required for efficient information processing in neural circuits (Rubenstein and Merzenich, 2003; Klausberger and Somogyi, 2008; Huang, 2009). The underlying components of E/I balance are the synaptic connections made between glutamatergic and GABAergic neurons. One key component to achieving proper E/I balance in a mature network is the maturation of GABAergic synapses (Grantyn et al., 2011).

Neuronal activity can affect synapse formation and function (Craig and Boudin, 2001; Burrone et al., 2002; Hartman et al.,

Received Jan. 16, 2013; revised Nov. 27, 2013; accepted Nov. 29, 2013.

Author contributions: C.-L.C., T.T., M.A.H., and C.R. designed research; C.-L.C., T.T., J.-C.J., and M.A.H. performed research; H.-T.C. contributed unpublished reagents/analytic tools; C.-L.C., T.T., J.-C.J., and M.A.H. analyzed data; C.-L.C., M.A.H., and C.R. wrote the paper.

This work was supported by the German Research Council (DFG Training Grant GRK1123 to C.-L.C. and DFG Collaborative Research Grant SFB665 to C.-L.C., J.-C.J., and C.R.), the Humboldt Foundation (to M.A.H.), the European Research Foundation (Grant SynVglut to C.R.), and the Excellence Cluster Neurocure Exc257 (to C.R.). We thank Annegret Felies, Berit Soehl-Kielczynski, Sabine Lenz, Katja Poetschke, and Bettina Brokowski for technical support; Dr. Thomas Mueller for supplying the GAD67-GFP $(\Delta$ neo)/+ mice; Dr. Yongling Zhu for supplying the SypH $2 X$ construct; Dr. Benjamin Rost for comments on the manuscript; and members of the Rosenmund laboratory for discussions.

The authors declare no competing financial interests.

Correspondence should be addressed to either Christian Rosenmund or Melissa A. Herman, CharitéUniversitätsmedizin Berlin, Charitéplatz 1, 10117 Berlin, Germany. E-mail: christian.rosenmund@charite.de or melissa.herman@charite.de.

DOI:10.1523/JNEUROSCI.0229-13.2014

Copyright $\odot 2014$ the authors $\quad 0270-6474 / 14 / 340855-14 \$ 15.00 / 0$
2006; Huang, 2009; Lu et al., 2009), which ultimately shapes the properties of a network. Glutamatergic input has been shown to affect GABAergic synapse number/function in an activitydependent manner (Marty et al., 2000; Colin-Le Brun et al., 2004; Hartman et al., 2006) and it has been proposed that activitydependent maturation of GABAergic neurons during development includes synaptogenesis (Marty et al., 2000; Chattopadhyaya et al., 2004; Colin-Le Brun et al., 2004) and decreased release probability characteristics (Morales et al., 2002; Tang et al., 2007; Jiang et al., 2010). Recent evidence suggests that eliminating postsynaptic transmission in individual excitatory neurons in vivo also decreases the strength of inhibitory transmission onto those neurons (Lu et al., 2013). Although there is ample evidence that synaptic input modifies GABAergic neurons, whether these changes simultaneously affect synapse formation, synapse output function, or other characteristics of GABAergic function is little understood.

To determine how glutamatergic input affects this set of parameters in GABAergic neurons in a quantitative manner, we chose a simplified circuit (Cohen and Segal, 2011; Williams et al., 2011) of glutamatergic-GABAergic two-neuron microcultures. As reported previously in culture preparations, glutamatergic synapse formation was not affected by activity (Craig et al., 1994; Rao and Craig, 1997; Gomperts et al., 2000; Burrone et al., 2002; Wierenga et al., 2006) or the presence of a paired neuron (Mennerick et al., 1995; Harms et al., 2005). On the contrary, we found 
that the GABAergic neuron increased its number of synaptic outputs in response to glutamatergic input while simultaneously decreasing its synaptic efficiency by way of decreased release probability. The modulation of GABAergic synapse formation and function by glutamatergic input was not target-cell specific with regard to an excitatory or inhibitory postsynaptic cell identity, but rather depended on activity in the glutamatergic neuron. In addition, we found the first evidence that glutamatergic neuronal activity altered glutamatergic input onto GABAergic neurons by changing AMPA receptor (AMPAR) properties. Together, our data suggest that glutamatergic input induces significant alterations of GABAergic neuron input-output function through multiple parameters. These findings may have implications in the proper formation of developing neural circuits, especially the maturation process of newly incorporated GABAergic neurons.

\section{Materials and Methods}

Neuronal culture. Murine microisland cultures were prepared as described previously (Xue et al., 2007). Hippocampal neurons from newborn mice (P0-P1) of either sex were plated at a density of 4000 neurons per $35-\mathrm{mm}$-diameter dish. We chose islands that contained a pair of neurons for recordings. Cells were used for electrophysiology and morphological analysis at $12-15$ days in vitro (DIV). For experiments in which neuronal activity was blocked, neurons were cultured in the presence of $0.5 \mu \mathrm{M}$ TTX (Tocris Bioscience) added at DIV 6, 10, and 14. For experiments in which ionotropic glutamatergic receptors were blocked, neurons were cultured in the presence of $2 \mu \mathrm{M}$ 2,3-dihydroxy-6-nitro-7sulfamoyl-benzo[f] quinoxaline-2,3-dione (NBQX; Tocris Bioscience) and $100 \mu \mathrm{M} \mathrm{D}-(-)-2$-amino-5-phosphonopentanoic acid (APV; Tocris Bioscience) or $10 \mu \mathrm{m}$ philanthotoxin-433 (Sigma-Aldrich) added at DIV 6,10 , and 14 .

In some experiments (Figs. 1, 2, 6, 7), we cultured neurons from GAD67-GFP $(\Delta$ neo $) /+($ GAD67) mice, in which GABAergic neurons expressing GAD67 are fluorescently labeled (Tamamaki et al., 2003). Comparison of electrophysiological response properties of striatal GABAergic GAD67-GFP ( $\Delta$ neo)/+ and wild-type neurons revealed no significant differences in synaptic properties: spontaneous miniature IPSCs (mIPSCs) amplitudes: WT; $43.6 \pm 2.6 \mathrm{pA}, n=30$; GAD67; $38.8 \pm$ $2.6 \mathrm{pA}, n=31 ; p>0.05$; vesicular release probabilities $\left(P_{\mathrm{vr}}\right)$ : wild-type (WT); $14.0 \pm 2.3 \%, n=14$; GAD67; $14.3 \pm 2.3 \%, n=11 ; p>0.05$.

Lentivirus constructs and production. For cell-type-specific expression of Kir2.1, we made use of the Cre/LoxP recombination system in combination with two lentiviral shuttle vectors and Viaat (VGAT)-promoterdriven expression of Cre recombinase (Viaat Cre mice; Chao et al., 2010). For glutamatergic expression, we used a floxed lentiviral shuttle vector in which a synapsin promoter controlled expression cassette of Kir2.1 fused to nuclear localization sequence-tagged green fluorescent protein (NLS-GFP) via a self-cleaving P2A peptide (Kim et al., 2011) is framed by two likewise-oriented mutant LoxP sites [LE/RE mutation ( $\mathrm{f}(\mathrm{syn})$ FLOX(NLS-GFP-P2A-Kir2.1)]. In GABAergic cells, Viaat-promoter-driven Cre expression causes the deletion of the NLSP2A-Kir2.1 transgene, resulting in no NLS-GFP or Kir2.1 expression (Albert et al., 1995; Araki et al., 1997). For expression of Kir2.1 in GABAergic neurons, we used a synapsin-promoter-controlled Crerecombinase-dependent lentiviral expression system carrying a reversed and double-floxed NLS-GFP-P2A-Kir2.1 transgene with two nested pairs of incompatible mutant (LE/RE) lox sites (LoxP and Lox2722). A red fluorescent protein (RFP) transgene was cloned in sense orientation between the first two lox sites [f(syn)LoxP_RFP_Lox2722_reverseNLSGFP-P2A-Kir2.1_LoxP_Lox2722]. In this configuration, RFP is only expressed in non-Cre-expressing cells. In Viaat-Cre GABAergic neurons, Cre expression leads to a switch from RFP to NLS-GFP and Kir2.1 translation due to excision and inversion events by Cre at the lox sites (Sohal et al., 2009). The resulting two lox sites are incompatible and doubly mutated to prevent further genomic recombination (Albert et al., 1995; Araki et al., 1997; Sohal et al., 2009). Control pairs for each of the cell- type-specific Kir2.1 expression groups were infected with these constructs lacking the sequence for Kir2.1.

For pHluorin-imaging experiments, the coding sequence of SypH $2 \mathrm{X}$ (Zhu et al., 2009) was PCR amplified and cloned in the lentiviral shuttle vector downstream of the synapsin promoter sequence using the restriction enzymes AscI and PacI.

After sequence verification, lentiviral particles were prepared as described previously (Lois et al., 2002). Briefly, HEK293T cells were cotransfected with $10 \mu \mathrm{g}$ of shuttle vector and the helper plasmids pCMVdR8.9 and pVSV.G (5 $\mu$ g each) with X-tremeGENE 9 DNA transfection reagent (Roche Diagnostic). After $72 \mathrm{~h}$, the virus-containing cell culture supernatant was collected and purified by filtration. Aliquots were flash-frozen in liquid nitrogen and stored at $-80^{\circ} \mathrm{C}$. For infection, virus $\left(6 \times 10^{5}\right.$ to $\left.1 \times 10^{6} \mathrm{IU} / \mathrm{ml}\right)$ was added to 1 DIV hippocampal neurons.

Electrophysiology. Whole-cell voltage-clamp recordings were performed with a patch-clamp amplifier (MultiClamp 700B amplifier; Molecular Devices) under the control of Clampex 9.2 (Molecular Devices). Data were acquired at $10 \mathrm{kHz}$ and low-pass filtered at $3 \mathrm{kHz}$. The holding potential was $-70 \mathrm{mV}$. The series resistance was compensated at $70 \%$ and only cells with series resistances $<10 \mathrm{M} \Omega$ were analyzed. The pipette resistance was between 2 and $5 \mathrm{M} \Omega$. The standard extracellular solution contained the following (in $\mathrm{mM}$ ): $140 \mathrm{NaCl}, 2.4 \mathrm{KCl}, 10 \mathrm{HEPES}, 10$ glucose, $2 \mathrm{CaCl}_{2}$, and $4 \mathrm{MgCl}_{2}$ (all from Carl Roth), $300 \mathrm{mOsm}, \mathrm{pH}$ 7.4. Hypertonic solution for measuring readily releasable vesicle pool (RRP) size was made by adding $500 \mathrm{~mm}$ sucrose (Sigma-Aldrich) to the standard extracellular solution. The $\mathrm{KCl}$ patch pipette solution contained the following (in mM): $136 \mathrm{KCl}, 17.8$ HEPES, and 1 EGTA (Carl Roth); 0.6 $\mathrm{MgCl}_{2}$ and 4 ATP-Mg (Sigma-Aldrich); 0.3 GTP-Na (Sigma-Aldrich); 12 phosphocreatine (Calbiochem, MERCK); and $50 \mathrm{U}^{*} \mathrm{ml}^{-1}$ phosphocreatine kinase (Sigma-Aldrich), $300 \mathrm{mOsm}$, pH 7.4. This solution set the reversal potential of IPSCs and EPSCs to $\sim 0 \mathrm{mV}$.

Step depolarization (to $0 \mathrm{mV}, 2 \mathrm{~ms}$ ) was applied sequentially (with $2 \mathrm{~s}$ delay) to each neuron to induce a single action potential in the respective axon, resulting in pairs of autaptic and heterosynaptic postsynaptic currents (PSCs). The RRP was determined as in Rosenmund and Stevens (1996).

In heterotypic pairs (glu-GABA), quantification of PSC, RRP, and $P_{\mathrm{vr}}$ was accomplished by consecutively blocking the glutamatergic and GABAergic contributions with kynurenic acid ( $3 \mathrm{~mm}$; Tocris Bioscience) and bicuculline (30 $\mu \mathrm{M}$; Tocris Bioscience), respectively. For all pairs, output PSCs were defined as the sum of autaptic and heterosynaptic PSCs. For heterotypic (glu-GABA) pairs, output RRPs were defined as the sum of the autaptic and heterosynaptic hypertonic sucrose-evoked responses. For homotypic (glu-glu or GABA-GABA) pairs, RRP sizes were defined as the sum of autaptic and heterosynaptic connections onto a neuron (input RRP), because the contribution of each neuron is not pharmacologically distinguishable in this configuration. Because amplitudes of output PSC and sizes of input RRP were comparable between the two neurons in glu-glu (output PSC: $p=0.78$; input RRP: $p=0.72$ ) or GABA-GABA pairs (output PSC: $p=0.20$; input RRP: $p=0.21$ ), the input RRP was assumed to be equivalent to output RRP in homotypic pairs.

Vesicular release probability $\left(P_{\mathrm{vr}}\right)$ was calculated by dividing the evoked response ( 1 or $2 \mathrm{~s}$ integration for EPSC or IPSC, respectively) charge by the RRP charge. In homotypic pairs, the mean input $P_{\mathrm{vr}}$ was defined as $P_{\mathrm{vr}}$ of autaptic and heterosynaptic connections ending at a common postsynaptic neuron and was calculated by dividing the sum of a neuron's input autaptic PSC charge and input heterosynaptic PSC charge from the partner neuron by its input RRP charge.

Paired-pulse ratios (PPRs) were computed by dividing the second PSC amplitude by the first (PPR = EPSC2/EPSC1 and IPSC2/IPSC1) of a pair of PSCs stimulated at a specific interval (EPSCs $25 \mathrm{~ms}$; IPSCs $100 \mathrm{~ms}$ ). Spontaneous release was detected as described previously (Clements and Bekkers, 1997).

For recording the current-voltage relationship of AMPAR-mediated EPSCs, the presynaptic neuron (glutamatergic) was stimulated by step depolarization with $\mathrm{KCl}$ patch pipette solution. Whole-cell currents were obtained in the postsynaptic neurons (glutamatergic or GABAergic) with 
a CsCl patch pipette solution containing the following (in mM): $143 \mathrm{CsCl}$; 20 HEPES; 1 EGTA (Carl Roth); $0.6 \mathrm{MgCl}_{2}$; 4 ATP-Mg (Sigma-Aldrich); 0.3 GTP-Na (Sigma-Aldrich); 12 Phosphocreatine (Calbiochem, MERCK); $50 \mathrm{U} \mathrm{ml}^{-1}$ Phosphocreatine kinase (Sigma-Aldrich); 2 QX314; 0.05 spermine ( $300 \mathrm{mOsm} ; \mathrm{pH} 7.4)$. APV $(100 \mu \mathrm{M})$ and bicuculline (30 $\mu \mathrm{M})$ were present in the extracellular solution to isolate AMPAR responses. Current-voltage plots were generated by plotting the EPSC amplitudes at holding potentials of -80 to $+60 \mathrm{mV}$ with $20 \mathrm{mV}$ increments. The current-voltage relationship in control and Kir2.1 expressing neurons in a glu-GABA pair were obtained by recording the current response to hyperpolarizing steps from -80 to -110 with $10 \mathrm{mV}$ decrements with $\mathrm{KCl}$ patch pipette solution.

Data were analyzed offline using AxoGraph X (AxoGraph Scientific), KaleidaGraph (Synergy Software), and Prism5 (GraphPad). Statistical significance was tested using the Student's $t$ test for two groups with normal distribution and one-way ANOVA with a Tukey post hoc test for three or more groups.

Immunocytochemistry and morphological analysis. Quantification of dendritic tree size and synapse type and number was performed using immunocytochemical staining for MAP2, VGLUT1, and VGAT. For relative expression levels of activity-dependent or cell growth markers, staining for c-fos or pS6, respectively, in glu-GABA microculture pairs cultured in the presence or absence of $0.5 \mu \mathrm{M}$ TTX (Tocris Bioscience) added at DIV 6, 10, and 14 was also used. Microisland cultures from wild-type mice were fixed at DIV $12-15$ with $4 \%$ paraformaldehyde (Sigma-Aldrich) and permeabilized with $0.02 \%$ Tween 20 in PBS (PBST). After blocking with 4\% normal goat serum (NGS; Jackson ImmunoResearch) in PBST for $1 \mathrm{~h}$ at room temperature, the specimens were incubated overnight at $4^{\circ} \mathrm{C}$ with guinea pig anti-VGLUT1 (1:4000; Synaptic Systems), mouse anti-VGAT (1:1000; Synaptic Systems), and chicken anti-MAP2 (1:2000; Millipore). In some experiments, rabbit anti c-fos (1:500; Abcam) or rabbit anti pS6 (1:500; Cell Signaling Technology) were included. After washing 3 times with PBST for $10 \mathrm{~min}$, cells were incubated for $1.5 \mathrm{~h}$ at room temperature in $4 \%$ NGS containing the appropriate secondary antibody (Alexa Fluor 488, 555, or 647 at 1:1000; Molecular Probes, Invitrogen). The specimens were then washed three times with PBST for 15 min and mounted on glass slides with Prolong Gold Antifade Reagent (Molecular Probes, Invitrogen). The fluorescence signals were visualized on an Olympus IX 81 microscope with a 20×, 0.75 numerical aperture (NA) objective lens and images were captured with a CCD camera (Princeton MicroMax; Roper Scientific).

Image analysis was performed using ImageJ. The type of neuron (GABAergic/glutamatergic) was identified by elevated fluorescence signals for VGLUT1 or VGAT at the cell soma (Hartman et al., 2006). For analysis of dendritic length and synapse number, the associated dendrites were determined by tracing using MAP2 staining. The density of synapses along three randomly chosen dendrites was determined by manual counting. Total dendritic length was quantified by measuring the length of all MAP2-positive processes using a custom-written plug-in in ImageJ (Neuron J). The total number of glutamatergic and GABAergic synapses was quantified by fluorescence signals for VGLUT1 and VGAT, respectively, using a custom macro in ImageJ. For assessing relative expression levels of c-fos and pS6, $z$-stack images with five sections were acquired and average intensity projections were calculated. From the projection image, the average intensity of the immunofluorescence signal for c-fos and pS6 in the nucleus and cytoplasm, respectively, was measured in ImageJ using custom macros and compared from both glutamatergic and GABAergic neurons with and without TTX treatment in glu-GABA pairs. All data processing was performed using Microsoft Excel, KaleidaGraph (Synergy Software), and Prism5 (GraphPad). Statistical significance was tested using Student's $t$ test for two groups with normal distribution and one-way ANOVA with a Tukey post hoc test for three or more groups.

Live cell imaging of SypH $2 X$ in pairs. Microisland cultures of hippocampal neurons were infected with lentivirus to express SypH $2 \mathrm{X}$ (Zhu et al., 2009). Pairs of neurons were patch-clamped as described in the electrophysiology method section. Neurons were consecutively stimulated with 300 action potentials (APs) at $20 \mathrm{~Hz}$ and corresponding 512 by 512 pixel images were captured at a rate of $1 \mathrm{~Hz}$ with a $20 \times$ objective (0.75 NA) and EMCCD camera (ImageEM; Hamamatsu) mounted on an inverted microscope (IX 51; Olympus). The neurotransmitter type of each neuron was determined by the kinetics of its postsynaptic response.

Images were analyzed using ImageJ. Image stacks were normalized to average baseline fluorescence (three images) before the start of stimulation. Two baseline-normalized images were averaged immediately after stimulation to generate the maximum $300 \mathrm{AP} \Delta F / F_{0}$ image. For the total area (pixels) of activated synapses measurement, a minimum threshold $\left(\Delta F / F_{0}=0.05\right)$ for the maximum $300 \mathrm{AP} \Delta F / F_{0}$ image was set and the area of all pixels above this threshold was calculated. Significance of differences between the two groups of GABAergic neurons was tested using Student's $t$ test.

\section{Results}

\section{Basic synapse properties in mixed glutamatergic-GABAergic pairs}

To determine how synaptic input affects a neuron's output, we performed a systematic electrophysiological (functional) and morphological analysis of pairs of hippocampal neurons grown on a glial microisland (see Materials and Methods). In the case of heterotypic pairs, one cell is excitatory, releasing glutamate (glu), and the other is inhibitory, releasing GABA. In this network, four types of synaptic connections exist; excitatory autapses $\left(E_{\mathrm{A}}\right)$, excitatory heterosynapses $\left(E_{\mathrm{H}}\right)$, inhibitory autapses $\left(I_{\mathrm{A}}\right)$, and inhibitory heterosynapses $\left(I_{\mathrm{H}}\right)$ (Fig. $\left.1 A\right)$. To examine the specific influence of glutamatergic and GABAergic innervation on synaptic input and output, we compared heterotypic pairs to pairs in which both neurons released the same neurotransmitter (homotypic gluglu or GABA-GABA pairs; Fig. 1B). We quantified the strength of each connection using electrophysiological methods by measuring PSCs obtained by action potential induction (Fig. $1 C, D$ ). In addition, we measured the RRP size obtained by hypertonic sucrose application (Fig. 1C,D; Rosenmund and Stevens, 1996), which is the product of the RRP of individual synaptic terminals and the number of synapses. For heterotypic pairs, we consecutively applied the glutamatergic receptor antagonist kynurenic acid and the $\mathrm{GABA}_{\mathrm{A}}$ receptor antagonist bicuculline $(3 \mathrm{mM}$ and $30 \mu \mathrm{M}$, respectively; Fig. $1 C)$ to isolate IPSCs and EPSCs, respectively.

We found that glutamatergic neurons had comparable synaptic output in homotypic and heterotypic pairs as the output EPSCs $\left(E_{\mathrm{A}}+E_{\mathrm{H}}\right)$ and RRP sizes $\left(\mathrm{RRP}_{\text {glu }}\right)$ were unchanged (Fig. $1 C-E$; see Materials and Methods for details). In contrast, GABAergic neurons experienced a change in synaptic output when the pair was heterotypic as the output RRP size $\left(\mathrm{RRP}_{\mathrm{GABA}}\right)$ for GABAergic neurons increased to $250 \pm 30 \%$ of that measured for individual GABAergic neurons in homotypic pairs (Fig. 1E). This suggests a significant expansion in the number of vesicles available for release from GABAergic neurons receiving glutamatergic input. Interestingly, the AP-evoked IPSC responses $\left(I_{\mathrm{A}}+\right.$ $I_{\mathrm{H}}$ ) were not affected when the pair was heterotypic (Fig. 1E).

We found that the amplitudes of the PSC in homotypic pairs and both the EPSC and RRP in glutamatergic neurons from heterotypic pairs produced by the autaptic connections were on average larger than those produced by the heterosynaptic connections (Fig. $1 F$ ). This suggests that, at the least, there is no active suppression of autaptic synapse formation in our system. However, for GABAergic neurons in heterotypic pairs, the IPSC and RRP produced by the autaptic connections were not larger than those produced by the heterosynaptic connections (Fig. $1 F$ ). Whether this indicates a preference of GABAergic neuron to form synapses onto a glutamatergic partner will be further tested by morphological analysis (see Fig. 5). 
A

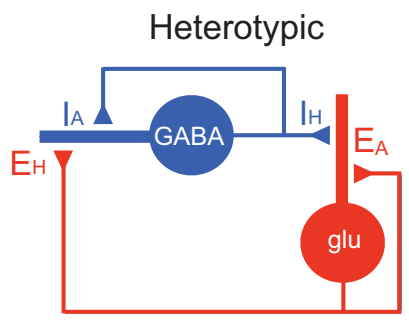

GABA output $=\mathrm{I}_{\mathrm{A}}+\mathrm{I}_{\mathrm{H}}$ glu output $=E_{A}+E_{H}$
C

\section{1. + kynurenic acid}

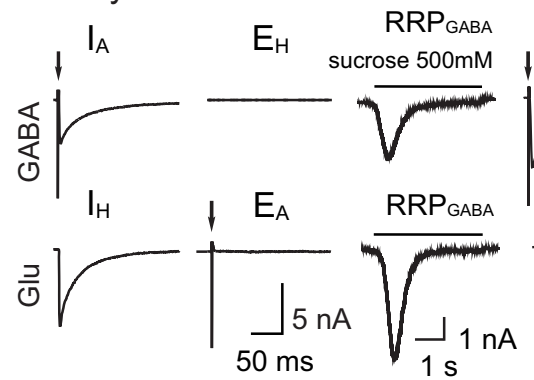

2. + bicuculline
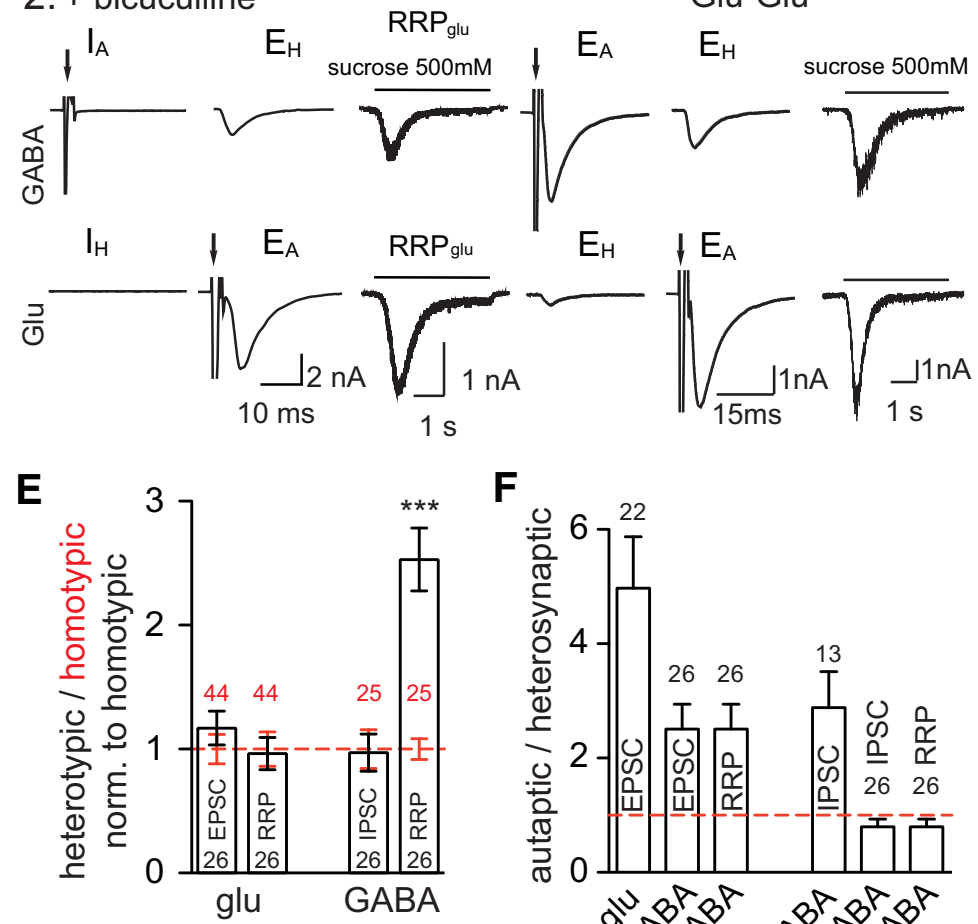

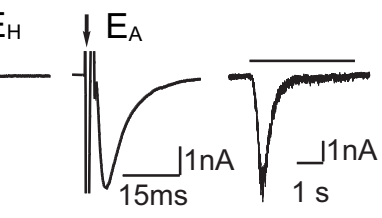

B

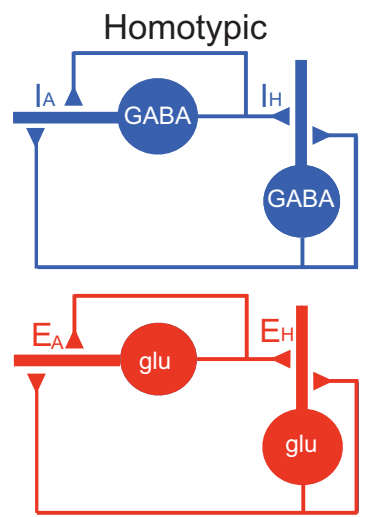

D

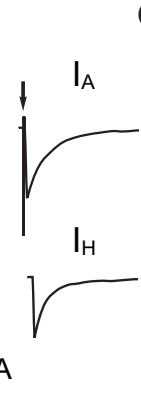

GABA-GABA

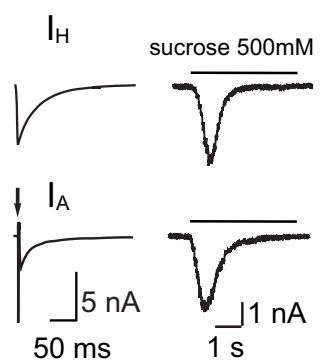

Glu-Glu

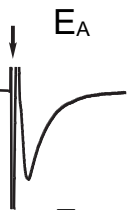

$F$

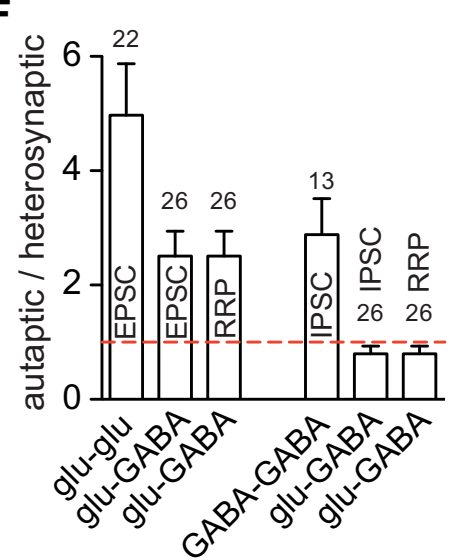

Figure 1. GABAergic output is modulated by glutamatergic input in glu-GABA pairs. $A$, Schematic diagram illustrating four different synaptic connections in a glu-GABA (heterotypic) neuronal circuit. The axon from a GABAergic or a glutamatergic neuron makes synapses onto itself (autaptic; $A$ ) and the partner neuron (heterosynaptic; $H$ ). The connections are as follows: Inhibitory autaptic connection $\left(I_{A}\right)$, inhibitory heterosynaptic connection $\left(I_{H}\right)$, excitatory autaptic connection $\left(E_{A}\right)$, and excitatory heterosynaptic connection $\left(E_{H}\right)$. $\boldsymbol{B}$, Schematic diagram illustrating two different synaptic connections for a GABA-GABA (homotypic) and a glu-glu (homotypic) pair. The connections in GABA-GABA neuronal pairs are $I_{\mathrm{A}}$ and $I_{\mathrm{H}}$. The connections in glu-glu neuronal pairs are $E_{\mathrm{A}}$ and $E_{\mathrm{H}} \cdot \boldsymbol{C}$, Representative traces of evoked IPSCs and EPSCS and sucrose responses from paired recording of a heterotypic neuron
Glutamatergic input modulates the efficiency of GABAergic synapses

Since glutamatergic innervation increases the $\mathrm{RRP}_{\mathrm{GABA}}$ size with no change in IPSC output, this may suggest a parallel reduction in efficiency of synaptic release. We probed this by measuring the vesicle release probability $\left(P_{\mathrm{vr}}\right)$ and paired-pulse ratios (PPRs) of glutamatergic and GABAergic neurons in glu-GABA pairs, glu-glu, and GABA-GABA pairs (Fig. 2). Indeed, we found that the GABAergic synapses in glu-GABA pairs displayed significantly lower $P_{\mathrm{vr}}$ and higher PPR compared with GABA-GABA pairs $\left(P_{\mathrm{vr}}\right.$ : GABA-GABA $20.6 \pm 1.6 \%$; glu-GABA $9.7 \pm 0.6 \%$; $p<0.0001$; PPR: GABAGABA $0.49 \pm 0.02$; glu-GABA $0.72 \pm$ $0.02 ; p<0.0001$; Fig. $2 A, B$ ). Conversely, in glutamatergic neurons, the output $P_{\mathrm{vr}}$ and PPR values were comparable in either glu-glu or glu-GABA pairs $\left(P_{\mathrm{vr}}\right.$ : glu-glu $5.9 \pm 0.5 \%$; glu-GABA $6.8 \pm 0.5 \%$; $p=$ 0.24 ; PPR: glu-glu $1.32 \pm 0.04$; glu-GABA $1.27 \pm 0.03 ; p=0.23$; Fig. $2 A, B)$. This suggests that although glutamatergic innervations to a GABAergic neuron may increase the total number of vesicles available for release, it decreases the release probability for each vesicle.

To determine whether the decrease in GABAergic synaptic efficiency in the gluGABA pair is target-cell specific, we compared the $P_{\mathrm{vr}}$ and PPR of GABAergic output onto the GABAergic postsynaptic cell $\left(I_{\mathrm{A}} ;\right.$ Fig. $\left.1 \mathrm{~A}\right)$ and onto the glutamatergic postsynaptic cell $\left(I_{\mathrm{H}}\right.$; Fig. $\left.1 A\right)$ in gluGABA pairs. We found that $P_{\mathrm{vr}}$ and PPR values were not different (Fig. 2D), demonstrating target cell indifferent behavior. Glutamatergic autapses and heterosynapses $\left(E_{\mathrm{A}}, E_{\mathrm{H}} ;\right.$ Fig. $\left.1 A\right)$ also showed similar $P_{\mathrm{vr}}$ and PPR values within glu-GABA pairs (Fig. 2D). To extend this analysis to ho-

\footnotetext{
$\leftarrow$

pair showing the ready releasable pool of GABAergic and glutamatergic vesicles (RRP ${ }_{\text {GABA }}$ and RRP glu, respectively). Responses were recorded from autaptic and heterosynaptic connections of each cell. GABAergic output was measured in the presence of kynurenic acid (C1) and glutamatergic output was determined in the presence of bicuculline (C2). Arrow indicates $2 \mathrm{~ms}$ somatic depolarization. $\boldsymbol{D}$, Representative traces of evoked IPSCS and EPSCS and sucrose responses from paired recordings of GABA-GABA and glu-glu homotypic pairs. Arrow indicates $2 \mathrm{~ms}$ somatic depolarization. $\boldsymbol{E}$, Bar graph showing the mean output PSC amplitudes and output RRP charges measured in heterotypic (black) and homotypic (red) neuronal pairs both normalized to homotypic values per culture. All values are mean \pm SEM. ${ }^{* * *} p \leq 0.001$. F, Bar graph showing the mean ratio of autaptic over heterosynaptic PSC and RRP responses of glutamatergic and GABAergic neurons in glu-GABA or homotypic neuronal pairs. Numbers in bar graphs are $n$ values.
} 
A
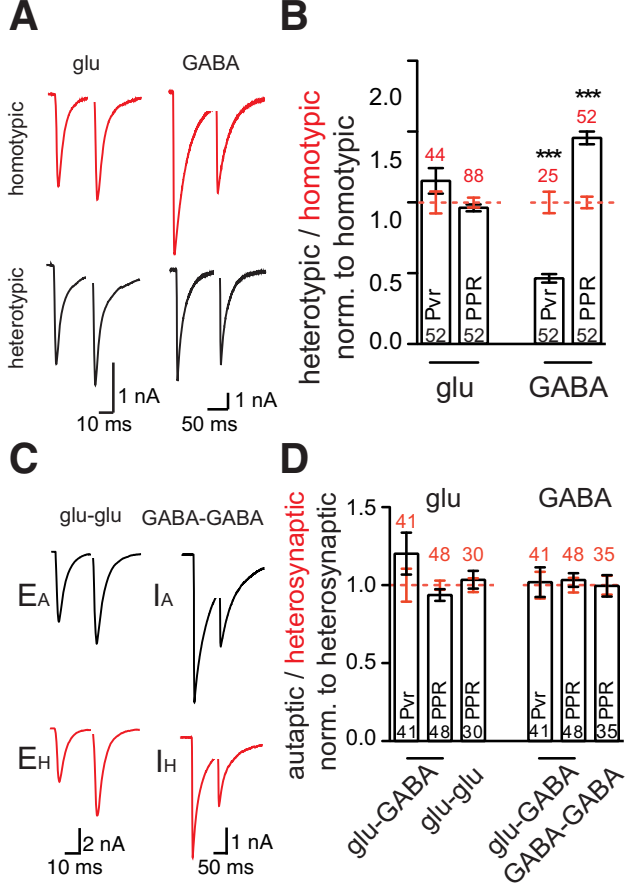

Figure 2. Glutamatergic input affects GABAergic synaptic efficiency in glu-GABA pairs. $\boldsymbol{A}$, Representative traces of paired-pulse EPSC (left) and IPSC (right) traces from heterosynaptic connections in homotypic (glu-glu, GABA-GABA; red traces) and heterotypic (glu-GABA; black traces) pairs. $\boldsymbol{B}$, Bar graph showing the $P_{\mathrm{vr}}$ and PPR in either glutamatergic or GABAergic neurons in glu-GABA (black) and homotypic (red) pairs normalized to the mean value from homotypic pairs per culture. ${ }^{* * *} p \leq 0.001$. C, Representative traces of paired-pulse EPSC (left) and IPSC (right) traces from autaptic (black) and heterosynaptic (red) connections in homotypic (glu-glu, GABA-GABA) pairs. D, Bar graph showing the autaptic (black) and heterosynaptic (red) PPR and $P_{\mathrm{vr}}$ in glu-GABA and homotypic neuronal pairs normalized to the heterosynaptic values. All values are mean \pm SEM. Numbers in bar graphs are $n$ values.

motypic pairs (both glu-glu and GABA-GABA), we found that PPRs were not different between autaptic and heterosynaptic connections (Fig. 2C,D). These data suggest that the decrease in synaptic release efficiency that occurs in GABAergic synapses in response to glutamatergic input happens in a cell-wide manner and is not target-cell specific, at least with regard to excitatory or inhibitory postsynaptic targets.

\section{Increased GABAergic synapse number contributes to increased RRP size}

The larger output RRP sizes of GABAergic neurons with glutamatergic innervation may suggest proliferation of GABAergic synapses. To test this, we compared the total synapse numbers using immunocytochemical methods (Fig. 3). We labeled markers for glutamatergic synapses and GABAergic synapses (VGLUT1 and VGAT, respectively; Fig. 3A). In isolated glutamatergic neurons, the glutamatergic synapse number was comparable to the number of glutamatergic synapses in glu-GABA pairs and the synapse number per cell in glu-glu pairs (total number of VGLUT1-positive synapses divided by two; Fig. $3 B$ ). In contrast, the number of GABAergic synapses in glu-GABA pairs was significantly larger than the synapse number of single GABAergic neurons (Fig. 3C). Additionally, there was a trend for the number of GABAergic synapses in glu-GABA pairs to be larger than the average number per cell in GABA-GABA pairs, though this difference did not reach signficance, likely due to each pair of GABA-GABA cells yielding only a single measurement (total number of VGAT-positive synapses divided by two; Fig. $3 C$ ).
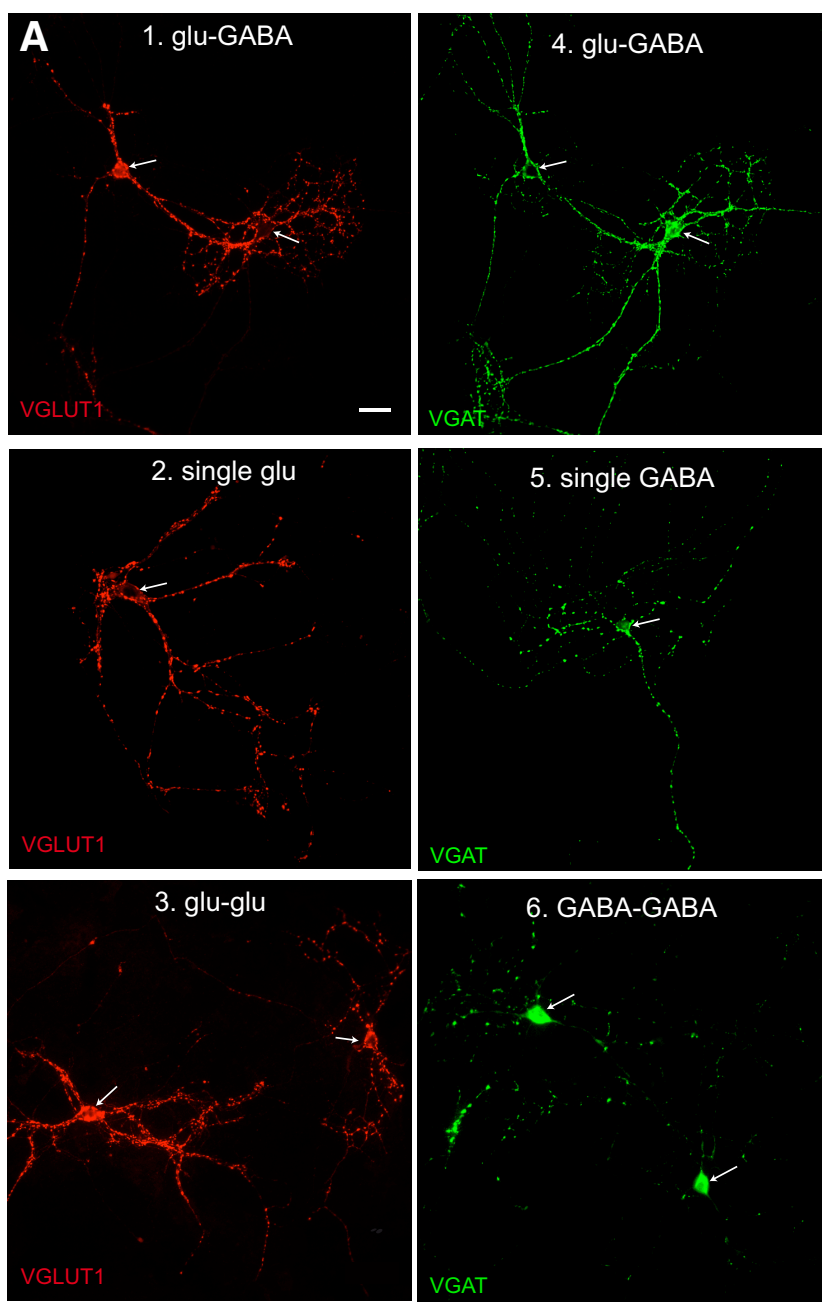

B

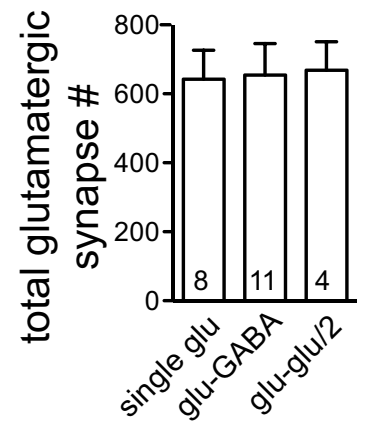

C

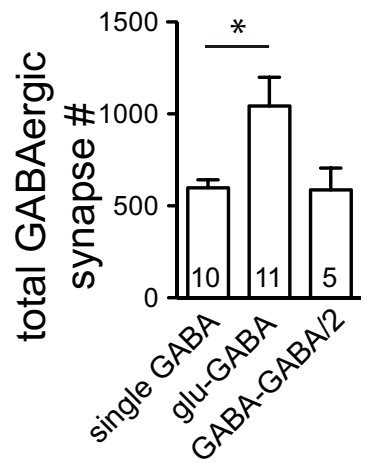

Figure 3. Glutamatergic input causes an increase in the number of GABAergic synapses. $\boldsymbol{A}$ Representative image of immunofluorescence for a glu-GABA neuronal pair $(\boldsymbol{A 1}, \boldsymbol{A 4})$, a single glutamatergic neuron (A2), a glu-glu neuronal pair (A3), a single GABAergic neuron (A5), and a GABA-GABA neuronal pair (A6) stained with glutamatergic (left; VGLUT1; red) and GABAergic (right; VGAT; green) synapse markers. Scale bar, $20 \mu \mathrm{m}$. Note that the type of neurons were apparent by the somatic VGLUT1 and VGAT signals (indicated by arrows). B, Quantification of total number of glutamatergic synapses (VGLUT1 positive) for isolated glutamatergic neuron, glu-GABA pair, or glu-glu pair (per cell; glu-glu/2). $\boldsymbol{C}$, Same as $\boldsymbol{B}$, but for the GABAergic cell type; ${ }^{*} p \leq 0.05$. All values are mean \pm SEM. Numbers in bar graphs are $n$ values.

This suggests that in the two-neuron microcircuit, a glutamatergic neuron makes the same total number of synaptic contacts independently of the postsynaptic neuron. However, the GABAergic neuron in a two-neuron microcircuit makes a higher number of total synaptic contacts with glutamatergic input. 


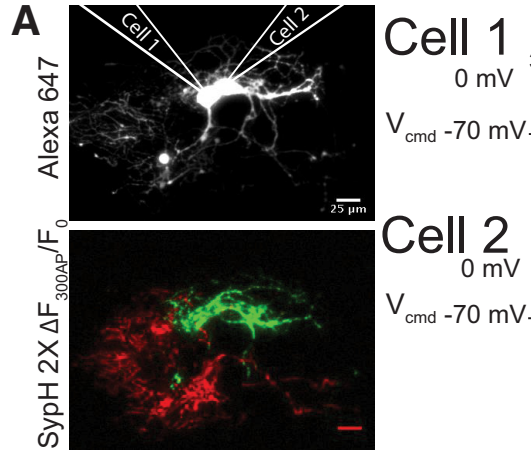

B
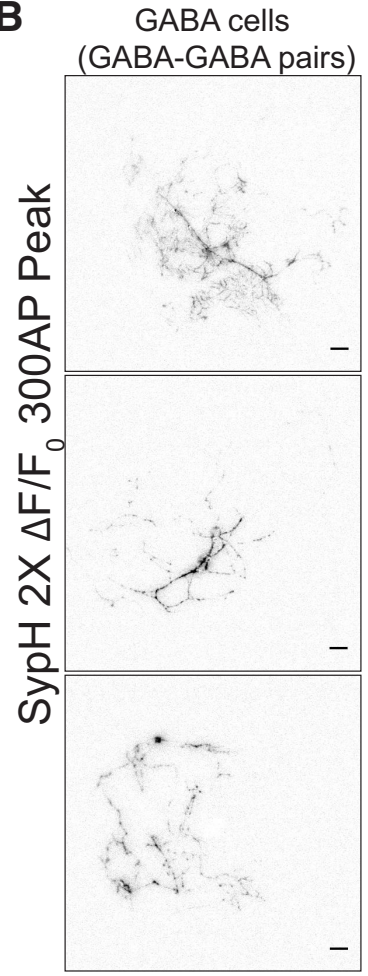

C

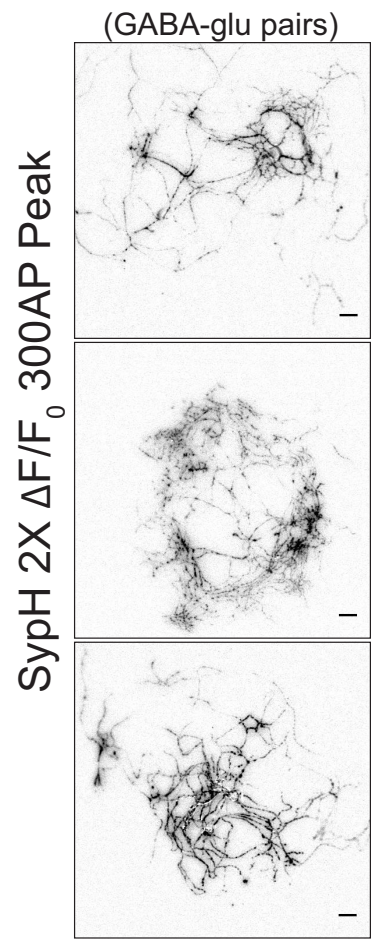

D

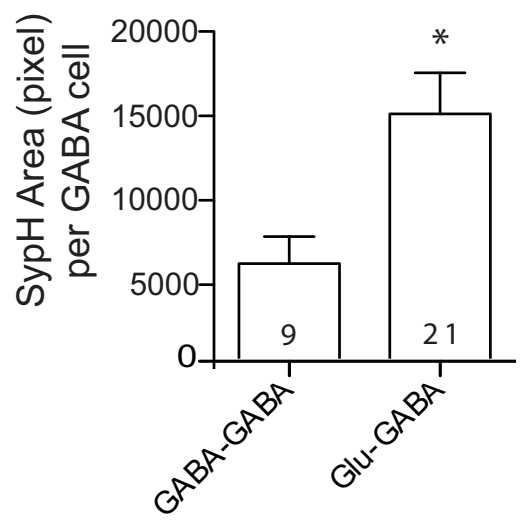

Figure 4. Glutamatergic input increases number of active synapses in GABAergic neurons. $\boldsymbol{A}$, Example image of a pair of neurons filled with intracellular Alexa Fluor 647 (100 $\mu$; t top left) in which each cell was stimulated with a $300 \mathrm{AP}$ train at $20 \mathrm{~Hz}$ consecutively (bottom left; scheme right). For illustrative purposes, SypH $2 \mathrm{X} \Delta F / F_{0}$ signal is represented in red for Cell 1 and green for Cell 2 (bottom left). The same color code is applied to the output PSCs (first 5 APs of 300 AP train) for each cell (right). Cell1 is GABAergic and Cell2 is glutamatergic. $B$, Examples of peak $\Delta F / F_{0}$ for SypH $2 X$ with a $300 \mathrm{AP}$ stimulation $(20 \mathrm{~Hz})$ in three individual cells from GABA-GABA pairs. Scale bar, $25 \mu \mathrm{m}$. Images are inverted for illustrative purposes and displayed on the same
To confirm that the increase in synapse number for GABAergic neurons in glu-GABA pairs as indicated by immunocytochemistry reflects synapses that actively undergo exocytosis and to account for the variability in GABAergic synapse number between cells in GABA-GABA pairs, we used an imaging approach to monitor presynaptic synapse activation (Fig. 4). In these experiments, a pair of neurons expressing synaptophysin tagged with two pHluorin proteins (SypH 2X; Zhu et al., 2009) was patched in whole-cell voltage-clamp mode and each cell was consecutively stimulated with 300 brief $(2 \mathrm{~ms}, 0 \mathrm{mV})$ depolarizations at $20 \mathrm{~Hz}$, which elicits an AP train at the same frequency (Fig. 4A). The neurotransmitter identity of each cell was determined by comparing the kinetics of the output PSC (Fig. 4A). We compared the area (in pixels) that showed an increase in SypH $2 \mathrm{X}$ fluorescence in response to the $300 \mathrm{AP}$ train $(20 \mathrm{~Hz})$ in GABAergic neurons from 21 glu-GABA pairs and five GABA-GABA pairs (Fig. $4 B, C$ ). We assume that the area of SypH $2 \mathrm{X}$ fluorescence is proportional to the number of synapses. Consistent with the immunocytochemistry analysis (Fig. 3) and RRP size measurements (Fig. 1), we found that the area of activated synapses in GABAergic neurons from glu-GABA pairs was $280 \pm 50 \%$ of that in GABAergic neurons from GABA-GABA pairs $(p=0.03$; Fig. $4 B-D)$. This suggests that GABAergic neurons generate more synapses when paired with a glutamatergic neuron than with another GABAergic cell.

\section{Glu-GABA pairs do not exhibit cell-type-dependent synapse formation}

To assess the target cell preference for the increased number of GABAergic synapses formed in glu-GABA pairs, we performed immunocytochemical experiments to compare the density of GABAergic synapses along dendrites of glutamatergic and GABAergic neurons (Fig. 5A, B; see Materials and Methods for details). In glu-GABA pairs, we found that glutamatergic neurons formed synapses onto themselves (glu autapse; Fig. $5 C$ ) or onto GABAergic neurons (glu hetero; Fig. $5 C$ ) with the same density (Fig. 5C). The density was approximately half of that from single glutamatergic neurons (Fig. $5 C$ ), consistent with the finding that the total number of synapses made by a glutamatergic neuron was not altered when a partner GABAergic neuron was present (Fig. $3 B$ ). In addition, GABAergic neurons in glu-GABA pairs formed synaptic contacts at the same density onto themselves (GABA autapse; Fig. $5 D$ ) or onto glutamatergic neurons (GABA hetero; Fig. 5D). However, the density was not reduced compared with that in single GABAergic neurons (Fig. 5D), which is consistent with the finding that a GABAergic neuron exhibited expansion of total synapse number when a glutamatergic partner neuron was present (Fig. 3C). Together, these data indicate that the synapse proliferation experienced by GABAergic neurons in glu-GABA pairs is not postsynaptic-target-cell specific.

If GABAergic neurons in a heterotypic pair form synapses at the same density without target-cell specificity, then what accounts for the disparity in autaptic to heterosynaptic IPSC and RRP for this configuration versus the others (Fig. $1 F$ )? To investigate this, we measured the total dendritic length using MAP2 staining and found that glutamatergic neurons had longer dendrites than GABAergic neurons in glu-GABA pairs (Fig. 5E).

scale. $\boldsymbol{C}$, Same as $\boldsymbol{B}$, but cells are from glu-GABA pairs. $\boldsymbol{D}$, Average area of SypH $2 \mathrm{X} \Delta F / F_{0}$ activated above threshold for individual GABAergic neurons in glu-GABA and GABA-GABA pairs. ${ }^{*} p \leq 0.05$. Values are mean \pm SEM. Numbers in graph show $n$ values. 
A Glutamatergic dendrite

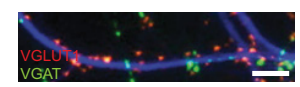

GABAergic dendrite
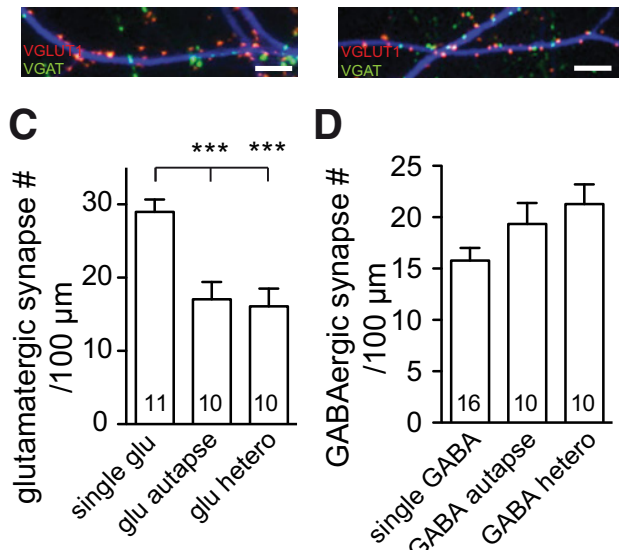

D

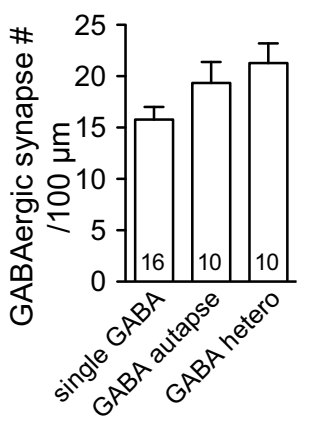

E

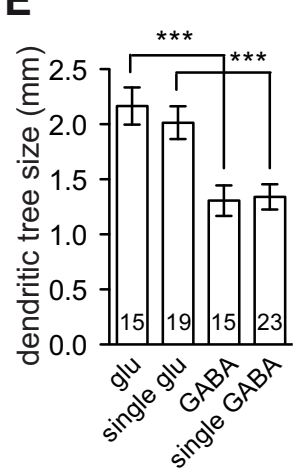

$\mathbf{F}$

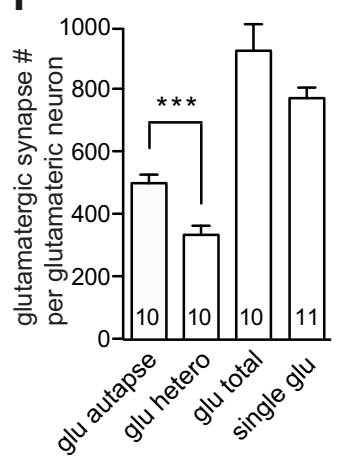

G

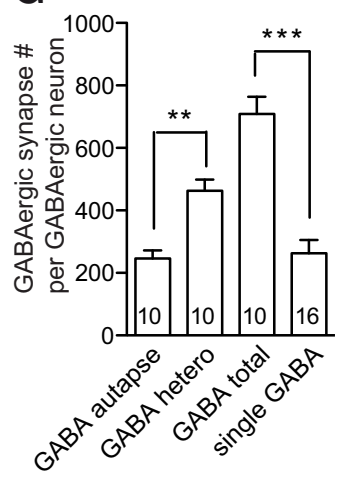

Figure 5. Glutamatergic input-induced increase in GABAergic synapse density. $\boldsymbol{A}, \boldsymbol{B}$, Representative images showing colocalization of VGLUT1 (red) and VGAT (green) with a dendritic marker, MAP2 (blue), immunofluorescence signals on identified glutamatergic $(\boldsymbol{A})$ and GABAe$\operatorname{rgic}(\boldsymbol{B})$ dendrites. VGLUT1 signals on the glutamatergic dendrite were considered autapses and VGAT signals were considered heterosynapses. On the GABAergic dendrite, VGAT signals were considered autapses and VGLUT1 signals were considered heterosynapses. Scale bar, $5 \mu \mathrm{m}$. C, Bar graph showing the mean synapse density (number of synapses per $100 \mu \mathrm{m}$ of dendritic length) of autapses formed in single glutamatergic neurons or autaptic and heterosynaptic connections of glutamatergic neurons in glu-GABA neuronal pairs. ${ }^{* *} p \leq 0.01$; ${ }^{* * *} p \leq 0.001$. $D$, Same as $C$, but for GABAergic neurons. $E$, Bar graph of the mean length of glutamatergic or GABAergic dendrites measured in a glu-GABA neuronal pair and single neurons as identified by the colocalization of MAP2 and either VGLUT1 or VGAT positive signals in the soma. $F$, Bar graph showing estimated number of VGLUT1-positive signals from glu-GABA neuronal pairs (autaptic connections, heterosynaptic connections, and total) and single glutamatergic neurons calculated from measurements in $\boldsymbol{C}$ and $\boldsymbol{E}$. $\boldsymbol{G}$, Same as $\boldsymbol{F}$, but for GABAergic synapse number calculated from measurements in $\boldsymbol{D}$ and $\boldsymbol{E}$. For all bar graphs, ${ }^{* *} p \leq 0.01 ;{ }^{* * *} p \leq 0.001$. All values are mean \pm SEM. Numbers in bar graphs are $n$ values.
These values were comparable to single neuron microislands (Fig. 5E). Using our density measurements along with the measured dendritic tree lengths, we again estimated the total number of synapses (Fig. $5 F, G$ ). From these measurements, we suggest that the disparity in autaptic to heterosynaptic response for GABAergic neurons in heterotypic pairs is likely due to differences in morphology of the two cell types (i.e., the longer dendrites in glutamatergic neurons) and is unlikely to be due to a postsynaptic cell-type-specific preference for formation of GABAergic synapses.

\section{Neuronal activity is required for the modulation of GABAergic synapse formation and function in glu-GABA pairs}

How do the inputs from glutamatergic neurons modulate the synapses of GABAergic neurons? One possible mechanism may involve increased neuronal activity, because this has been found to modulate glutamatergic and GABAergic synapse formation in mass culture preparations (Burrone et al., 2002; Hartman et al., 2006). To test this, we blocked neuronal activity with TTX $(0.5 \mu \mathrm{M})$ treatment of our cultures continuously from 6 to 7 DIV until recording (12-13 DIV). We found that TTX treatment blocked the effect of glutamatergic innervations on GABA synapse formation and function, because RRPGABA in TTX-treated glu-GABA pairs was similar to that in homotypic pairs (Fig. 6A,B). Furthermore, the GABAergic synapse density was significantly decreased compared with that in untreated pairs (synapses/100 $\mu \mathrm{m}$ dendrite length: untreated GABAergic autapse $33.5 \pm 3.7, n=8$, TTX-treated GABAergic autapse $12.9 \pm 1.6, n=15, p<0.001$; untreated GABAergic heterosynapse $33.0 \pm 3.0, n=8$, TTX treated GABAergic heterosynapse $20.3 \pm 1.9, n=15, p<0.001)$. The total number of synapses for a given presynaptic neuron was significantly decreased in GABAergic neurons, but not in glutamatergic neurons (Fig. 6C,D). Our findings are consistent with previous work showing that suppression of network activity is associated with a decreased density of GABAergic synapses in mass cultures (Hartman et al., 2006).

We also tested the effect of TTX treatment on the release probability of each neuron in glu-GABA pairs. After TTX treatment, the values of $P_{\mathrm{vr}}$ and PPR of GABAergic neurons paired with glutamatergic neurons were not significantly different from GABA-GABA pairs with or without TTX treatment (Fig. 6E-G). TTX treatment did not affect the $P_{\mathrm{vr}}$ of glutamatergic neurons in either glu-GABA or glu-glu pair configurations (nontreated, $P_{\mathrm{vr}}$ : glu-GABA: $6.2 \pm 0.6 \%, n=52$; glu-glu: $5.9 \pm 0.5 \%, n=14 ; p=$ 0.07; TTX: $P_{\mathrm{vr}}$, glu-GABA: $5.3 \pm 0.6 \%, n=48$; glu-glu: $5.0 \pm$ $0.7 \%, n=28 ; p=0.3$ ) or RRP, PPR, or $P_{\mathrm{vr}}$ of GABAergic neurons in GABA-GABA pairs (Fig. $6 A, B, F, G$ ).

Next, we decreased neuronal activity by blocking ionotropic glutamate receptors using NBQX $(2 \mu \mathrm{M})$ and APV $(100 \mu \mathrm{M})$ treatment during synapse development (from 6-7 DIV until recording at 12-13 DIV). Again, we found that GABAergic neurons in antagonist-treated glu-GABA pairs tended to display a decreased $\mathrm{RRP}_{\mathrm{GABA}}(p=0.08)$, whereas PPR decreased and $P_{\mathrm{vr}}$ increased significantly with respect to untreated pairs (Fig. $6 B, E-$ $G)$. We saw no change in the synapse properties of glutamatergic neurons (nontreated, $\mathrm{RRP}_{\text {glu }}: 0.97 \pm 0.1 \mathrm{nC}, n=26$; NBQX/ $\mathrm{APV}, \mathrm{RRP}_{\mathrm{glu}}: 1.23 \pm 0.1 \mathrm{nC}, n=20 ; p=0.28$; nontreated, $P_{\mathrm{vr}}$ : $6.2 \pm 0.6 \%, n=52$; NBQX/APV, $P_{\mathrm{vr}}: 5.15 \pm 0.9 \%, n=40 ; p=$ 0.23 ; nontreated, PPR: $1.30 \pm 0.1, n=48$; NBQX/APV, PPR: $1.32 \pm 0.1, n=28 ; p=0.90)$. Therefore, chronic activity blockade reversed the synaptic output phenotype of GABAergic neu- 
A

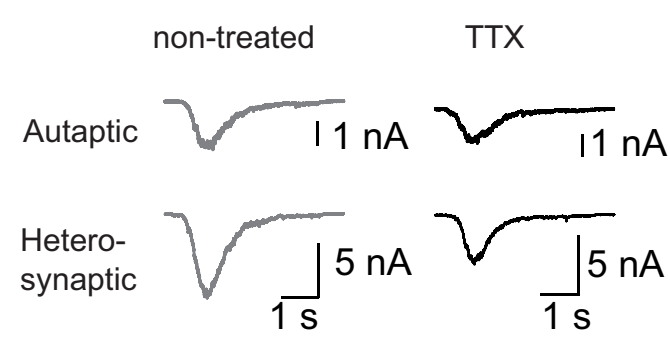

GABA-GABA

GABA-GABA TTX
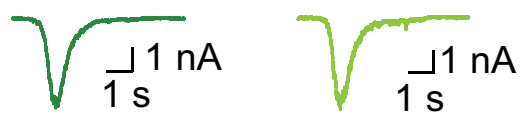

C
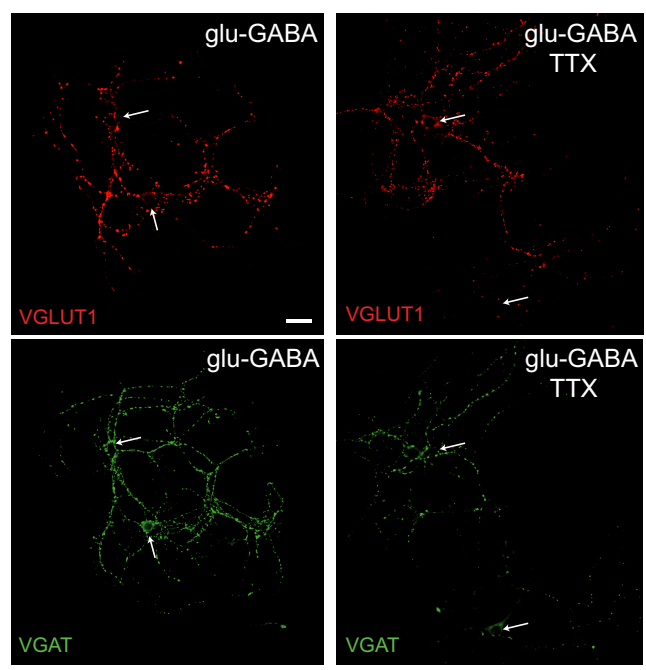

D
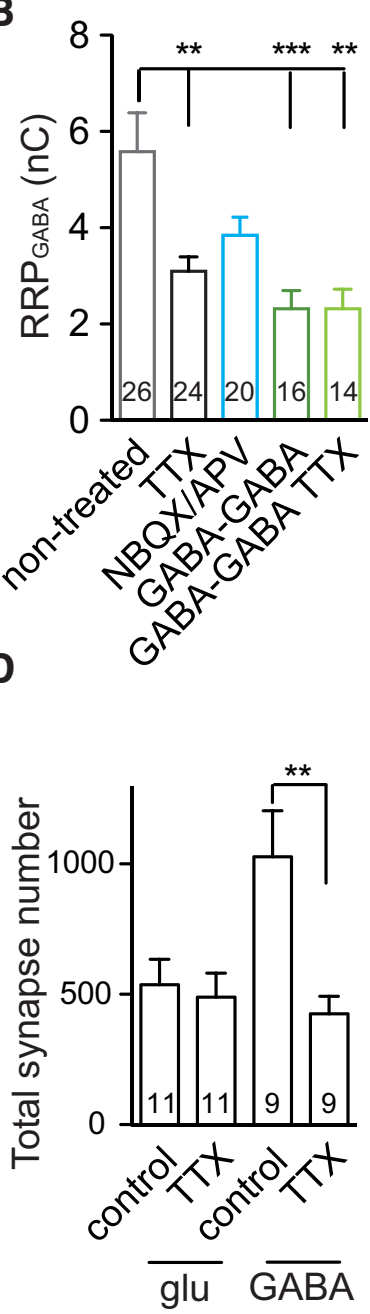

E

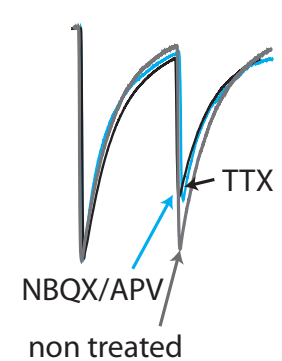

non treated

$50 \mathrm{~ms}$

G 30

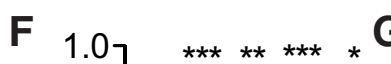

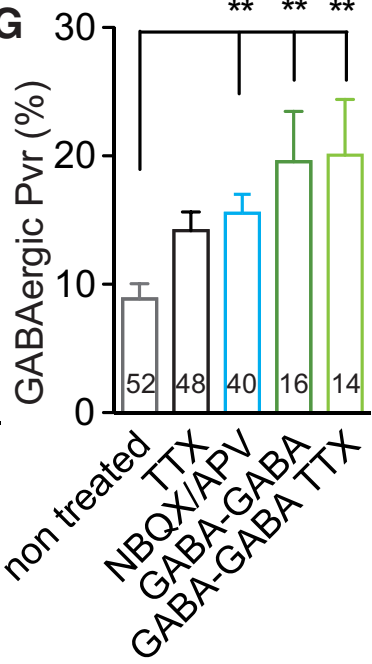

Figure 6. Activity modulates GABAergic synapse properties in glu-GABA pairs. $A$, Representative traces of GABAergic sucrose responses from paired recording of glu-GABA (top) and GABA-GABA (bottom) neuronal pairs with (black and light green, respectively) and without (gray and dark green, respectively) TTX treatment. Responses were recorded from autaptic and heterosynaptic connections of each GABAergic cell in glu-GABA neuronal pairs and from each GABAergic cell in GABA-GABA neuronal pairs. $\boldsymbol{B}$, Bar graph showing the mean $R_{R P} P_{G A B A}$ charges measured in glu-GABA pairs without (gray) and with TTX (black) or NBQX/APV (blue) treatment and GABA-GABA pairs with (light green) and without (dark green) TTX treatment. ${ }^{* *} p \leq 0.01 ;{ }^{* * *} p \leq 0.001$. $C$, Representative images of immunofluorescence for glu-GABA neuronal pairs stained with glutamatergic (top; VGLUT1; red) and GABAergic (bottom; VGAT; green) synapse markers without (left) and with (right) TTX. Note that the types of neurons were

rons in glu-GABA pairs to the phenotype of GABA-GABA pairs. Furthermore, the lack of changes in synaptic output in glutamatergic neurons upon treatment with TTX and ionotropic glutamate receptor antagonists suggests that glutamatergic synapse formation does not require neuronal activity/synaptic transmission, as suggested by previous studies (Craig et al., 1994; Rao and Craig, 1997; Gomperts et al., 2000; Verhage et al., 2000; Varoqueaux et al., 2002; Lu et al., 2013).

Specific inhibition of GluA2-lacking AMPARs with philanthotoxin-433 treatment during development $(10 \mu \mathrm{M}, 6 \mathrm{~d}$ before recording) also impaired the GABAergic output modification by decreasing $\mathrm{RRP}_{\mathrm{GABA}}$ sizes and increasing synaptic efficiency (data not shown; see Discussion). This indicates that GABAergic synaptic output modification in glu-GABA pairs requires neuronal activity and ionotropic glutamatergic receptor activation.

We next investigated whether neuronal activity alters gene expression in GABAergic neurons in glu-GABA pairs. Using immunocytochemistry, we examined the expression of an activitydependent marker (c-fos; Morgan and Curran, 1991; Ghosh et al., 1994; Flavell and Greenberg, 2008) and a cell growth marker (pS6; Flavell and Greenberg, 2008; Meyuhas, 2008; Knight et al., 2012) in identified glu-GABA pairs in the presence and absence of TTX. However, we saw no obvious changes for the markers tested in either cell type with TTX treatment (data not shown).

Neuronal activity is required for the modulation of glutamatergic input onto GABAergic neurons in glu-GABA pairs

Are postsynaptic response properties also altered in glu-GABA pairs? To test this, we examined mPSCs, which provide insight into postsynaptic receptor composition/ function. Autaptic mEPSCs in glu-GABA pairs were similar to mEPSCs in glu-glu

\section{$\leftarrow$}

apparent by the somatic VGLUT1 and VGAT signals (indicated by arrows). Scale bar, $20 \mu \mathrm{m}$ (D) Bar graph showing the total number of glutamatergic and GABAergic synapse in glu-GABA pairs without (control) and with TTX treatment. ${ }^{* *} p \leq 0.01 . E$, Representative paired-pulse IPSC traces recorded from glutamatergic neurons in untreated (gray), TTX-treated (black), and NBQX/APV-treated (blue) glu-GABA pairs. $F, G$, Bar graph showing the mean values of GABAergic PPR $(\boldsymbol{F})$ and $P_{\mathrm{vr}}(\boldsymbol{G})$ measured in glu-GABA pairs without (gray) and with (black) TTX or NBQX/APV (blue) treatment and GABA-GABA pairs with (light green) and without (dark green) TTX treatment. ${ }^{*} p \leq$ $0.05 ;{ }^{* *} p \leq 0.01 ;{ }^{* * *} p \leq 0.001$. All values are mean \pm SEM. Numbers in bar graphs are $n$ values. 
A
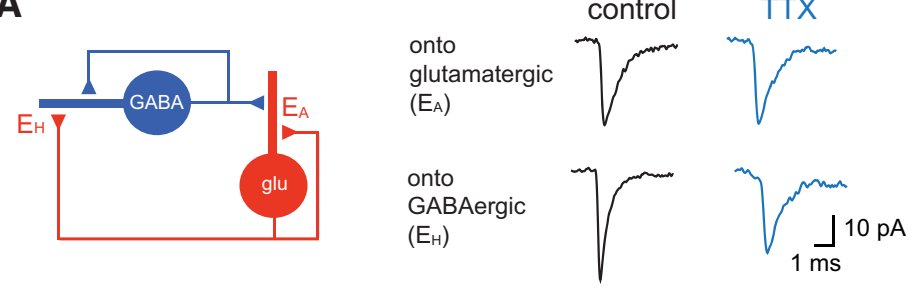

B

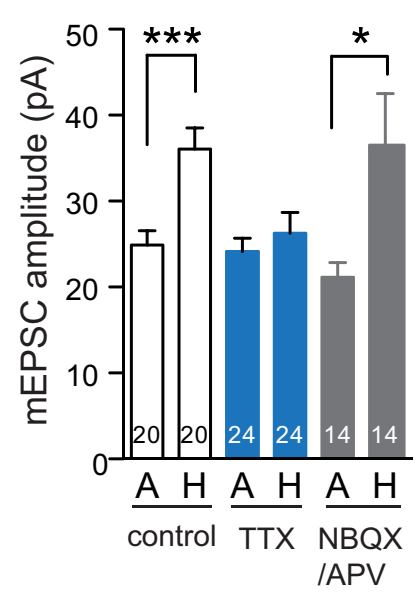

D

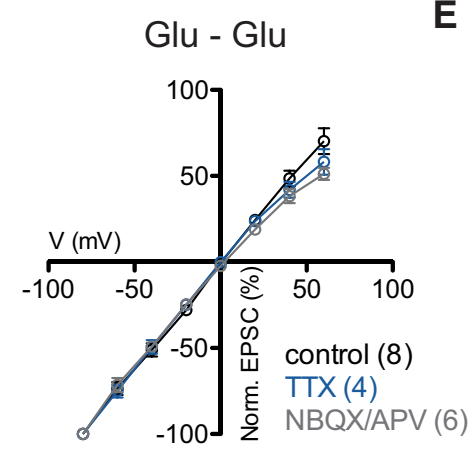

E

C
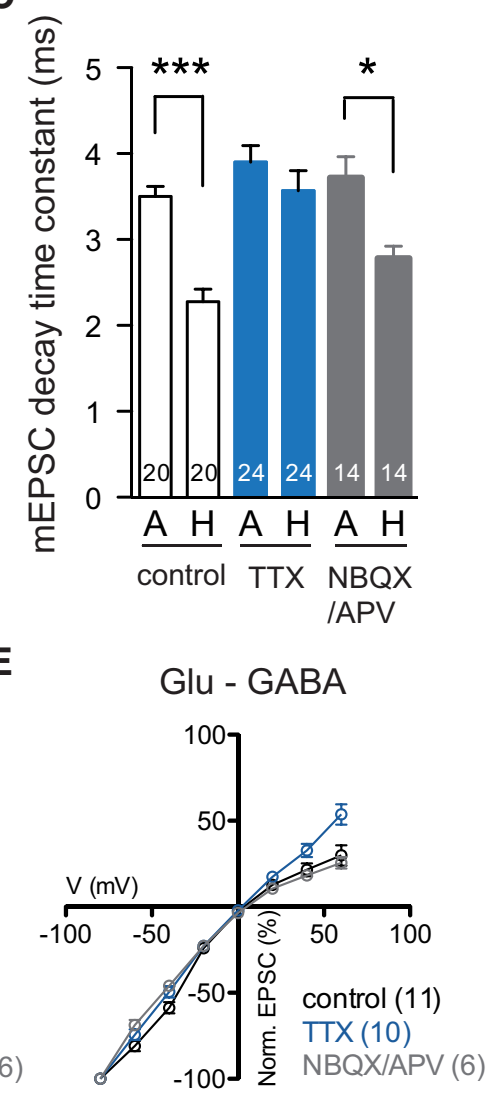

F

G

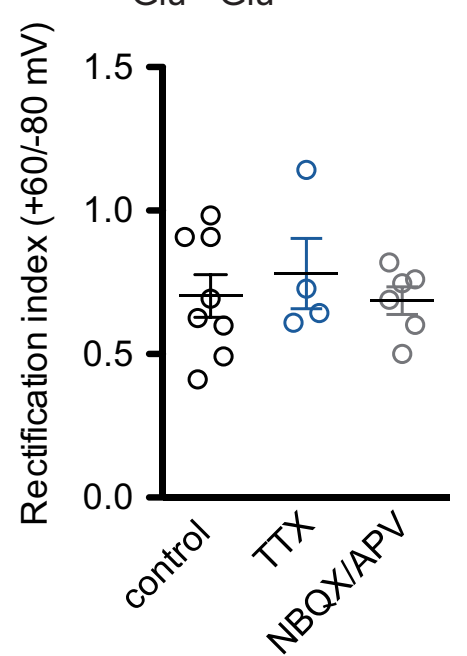

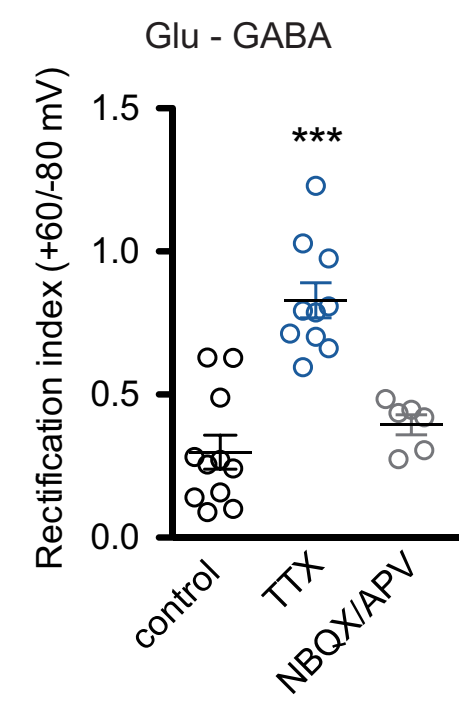

Figure 7. Activity-dependent modulation of glutamatergic input onto GABA neurons. $\boldsymbol{A}$, Left, Scheme of autaptic (A) and heterosynaptic $(H)$ connections made by a glutamatergic neuron in a glu-GABA pair. Right, Representative traces of mEPSCs recorded from glutamatergic (A: glutamatergic autapse; top) and GABAergic (H: glutamatergic heterosynapse; bottom) neurons without TTX (control, black) and with TTX (blue) treatment. $\boldsymbol{B}$, Bar graph showing the mean mEPSC amplitudes measured in

pairs $(25.7 \pm 1.5 \mathrm{pA}, n=20$ and $24.6 \pm$ $1.6 \mathrm{pA}, n=34$, respectively; $p=0.36$ ). However, mEPSCs onto GABAergic neurons showed significantly increased amplitudes (Fig. $7 A, B$ ) and faster decay kinetics (Fig. 7C), effects that could be due to a different AMPAR composition in GABAergic neurons, presumably including the GluA2-lacking AMPAR (Geiger et al., 1997). The mIPSCs in glu-GABA pairs were similar in amplitude regardless of the postsynaptic neuron identity (autaptic: $41.8 \pm 5.4 \mathrm{pA}, n=20$; heterosynaptic: $37.9 \pm 4.3 \mathrm{pA}, n=20 ; p>0.05)$. Furthermore, the kinetics of mIPSCs were not altered because the decay time constant was not significantly different between the two groups (data not shown).

Interneurons have been shown to express GluA2-lacking AMPARs in situ (Geiger et al., 1995; Isaac et al., 2007). However, it is undetermined whether this is due to a cell autonomous program that is set up in early development or if it is neuronal activity dependent. To test this, we studied the effect of chronic activity blockade on glutamatergic input onto GABAergic neurons in our two-cell culture system. TTX treatment (DIV 6-12) led to a significant decrease of mEPSC amplitudes onto GABAergic neurons (Fig. $7 A, B$ ) and slowing of the mEPSC decay time constant (Fig. $7 A, C$ ), reaching values similar to those seen in the other glutamatergic synapses, which argues for a change in AMPAR composition. However, blocking ionotropic glutamate receptors did not change the properties of mEPSCs onto GABAergic neurons (Fig. $7 B, C$ ). Therefore, glutamatergic input modifies postsynaptic glutamate responses in GABAergic neurons in an

\section{$\leftarrow$}

glutamatergic $(A)$ and GABAergic $(H)$ neurons in glu-GABA pairs without (white) and with TTX (blue) or NBQX/APV (gray) treatment. ${ }^{*} p \leq 0.05 ;{ }^{* * *} p \leq 0.001$. C, Bar graph showing the mean $m E P S C$ decay time constant measured in glutamatergic $(A)$ and GABAergic $(H)$ neurons in glu-GABA pairs without (white) and with TTX (blue) or NBQX/APV (gray) treatment. ${ }^{*} p \leq 0.05 ;{ }^{* * *} p \leq 0.001$. D, Current-to-voltage relationship of the heterosynaptic connection in glu-glu pairs without (control) and with TTX or NBQX/APV treatment. $E$, Same as $\boldsymbol{D}$, but with the glutamatergic heterosynaptic connection to GABAergic neurons in glu-GABA pairs. All values are mean \pm SEM. Numbers in bar graphs are $n$ values. $\boldsymbol{F}$, Rectification index values of the heterosynaptic connection in gluglu pairs without (control) and with TTX or NBQX/APV treatment. Rectification index values are determined by the ratio of peak amplitude +60 and $-80 \mathrm{mV}$. ${ }^{* *} p \leq 0.001$. G, Same as $\boldsymbol{F}$, but with the glutamatergic heterosynaptic connection to GABAergic neurons in glu-GABA pairs. Symbols represent rectification index values for individual cells. Mean indicated by line (black) and error bars represent SEM. 
activity-dependent manner, which requires AP firing. In contrast, autaptic mEPSCs in glu-GABA pairs were not affected by neuronal activity blockade with TTX or ionotropic glutamate receptor antagonist treatment (Fig. $7 A-C$ ), nor were mIPSC amplitudes of either synapse type (TTX-treated autaptic: $37.4 \pm 1.8 \mathrm{pA}, n=$ 24; TTX-treated heterosynaptic: $43.4 \pm$ $2.5 \mathrm{pA}, n=24)$. The lack of change in autaptic mEPSC amplitude with activity blockade suggests our two-cell culture system is lacking homeostatic synaptic plasticity (Turrigiano et al., 1998; Turrigiano and Nelson, 2004).

Synapses containing GluA2-lacking AMPARs exhibit inwardly rectifying postsynaptic responses (Jonas and Burnashev, 1995). Therefore, to further characterize the differences in AMPAR composition on GABAergic and glutamatergic neurons, we compared EPSC rectification in glu-glu and glu-GABA pairs. In glu-glu pairs, the EPSC in glutamatergic neurons exhibited a linear current-to-voltage relationship (Fig. 7D). In glu-GABA pairs, the EPSC in GABAergic neurons was partially inward rectifying (Fig. $7 E$ ), indicating the expression of GluA2-lacking AMPARs on GABAergic neurons. This rectification was abolished when neuronal activity was blocked by TTX treatment but not by the ionotropic glutamate receptor antagonists NBQX/APV (Fig. 7E). This was quantified by comparing the EPSC amplitude recorded at $+60 \mathrm{mV}$ with that recorded at $-80 \mathrm{mV}$ per cell to establish a rectification index (RI; +60/-80 mV; Bats et al., 2012). The RI was significantly larger for glutamatergic to GABAergic connections in TTX-treated cells compared with control and NBQX/APV-treated cells (Fig. $7 G$ ), suggesting a decrease in the contribution of inward rectifying current in this condition. The RI was not different in glutamatergic to glutamatergic connections for any condition tested (Fig. $7 F$ ). Therefore, these data indicate that neuronal activity, but not the activation of ionotropic glutamate receptor, shapes the GABAergic neuron's response to glutamatergic input.

Further analysis of the mPSC events revealed that mEPSC frequency (in glu-GABA pairs) was unchanged by pharmacological treatment (TTX: $11.2 \pm 1.3 \mathrm{~Hz}, n=20$; no TTX: $15.1 \pm 1.8$ $\mathrm{Hz}, n=19, p=0.08)$, but, analogous to the modification of RRP size, the frequency of mIPSCs was significantly reduced in the TTX-treated group (TTX: $5.1 \pm 0.8 \mathrm{~Hz}, n=22$; no TTX: $9.1 \pm$ $1.4 \mathrm{~Hz}, n=22, p=0.01)$.

\section{Glutamatergic neuronal activity is required for the} modulation of GABAergic synapse formation and function As shown in Figure 6, inhibition of global neuronal activity resulted in a decreased GABAergic synapse number, along with
B
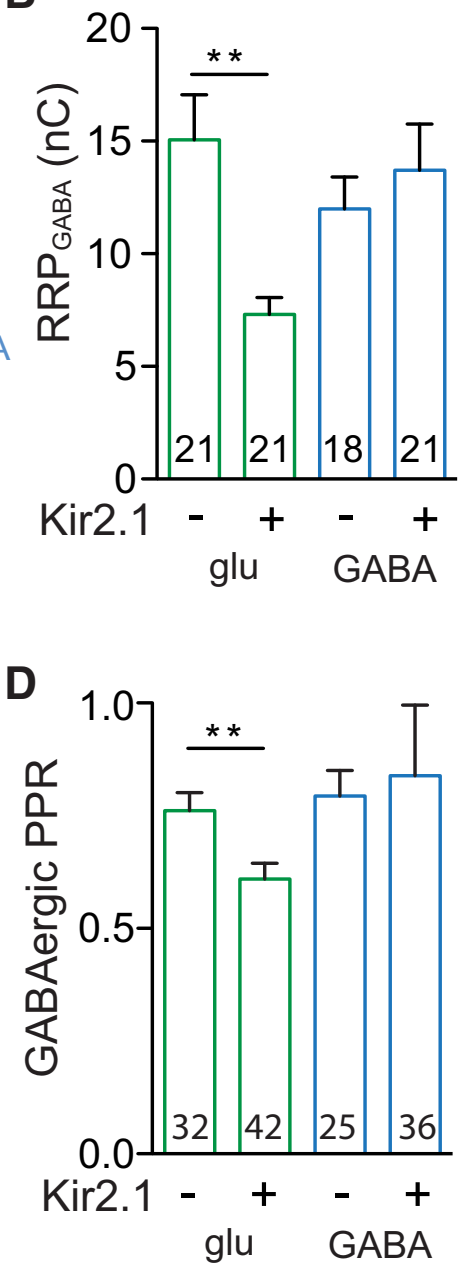

Figure 8. Suppression of glutamatergic neuronal activity by Kir2.1 prevents modulation of GABAergic output in glu-GABA pairs. $\boldsymbol{A}$, Top, Schematic representation and representative traces of GABAergic sucrose responses (green) from paired recordings of glu-GABA neuronal pairs where Kir2.1 was specifically expressed in the glutamatergic neuron. Bottom, Schematic representation nd representative traces of GABAergic sucrose responses (blue) from paired recordings of glu-GABA neuronal pairs in which Kir2.1 GABAergic connections. $\boldsymbol{B}$, Bar graph showing the mean $\mathrm{RRP}_{\mathrm{GABA}}$ charges (sum of $\mathrm{A}$ and $\mathrm{H}$ ) with either a Kir2.1 expressing $(+$ ) or control vector infected $\left(-\right.$ ) glutamatergic (green) or GABAergic (blue) neuron. ${ }^{* *} p \leq 0.01$. C, Top, Schematic representation and lutamatergic neuron. Bottom, Schematic representation and representative paired-pulse IPSC traces (blue) from glu-GABA neuronal pairs where Kir2.1 was specifically expressed in the GABAergic neuron. D, Bar graph showing the mean GABAergic PPR with either a Kir2.1-expressing $(+)$ or a control vector infected (-) glutamatergic (green) or GABAergic (blue) neuron. ${ }^{* *} p \leq 0.01$. All values are mean \pm SEM. Numbers in bar graphs are $n$ values.

increased synaptic efficiency in our two-neuron microcircuit. We aimed to study which cell type's activity is required to modulate GABAergic synaptic properties. By taking the advantage of our two-neuron culture system, we suppressed either glutamatergic or GABAergic neuronal activity by cell-type-specific overexpression of the inward-rectifying potassium channel Kir2.1 (see Materials and Methods; Burrone et al., 2002) and then examined the GABAergic synaptic output and input properties. Consistent with previous studies (Burrone et al., 2002), Kir2.1-expressing cells showed an inwardly rectifying current-to-voltage relationship (data not shown) and reduced input resistance $38 \pm 4.6 \%$ decrease in glutamatergic neurons; control, $n=18$, Kir2.1, $n=$ $12 ; 27 \pm 5.1 \%$ decrease in GABAergic neurons; control, $n=13$, Kir2.1, $n=14$. We found that if Kir2.1-expressing cells were glutamatergic, the $\mathrm{RRP}_{\mathrm{GABA}}$ sizes were significantly reduced 
compared with the control group (Fig. 8A,B). This was accompanied by increased synaptic efficiency, as indicated by a reduced PPR (Fig. $8 C, D$ ). However, these changes were not observed in pairs with a Kir2.1-expressing GABAergic neuron (Fig. 8A-D). Importantly, the glutamatergic neurons showed no change in output RRP or PPR regardless of whether they or the GABAergic neuron in the pair expressed Kir2.1 $\left(\mathrm{RRP}_{\mathrm{glu}}\right.$ : control $1.1 \pm 0.3 \mathrm{nC}$, $n=21$; Kir2.1 in glu $1.1 \pm 0.3 \mathrm{nC}, n=21 ; p=0.99$; control $1.0 \pm$ $0.2 \mathrm{nC}, n=18$, Kir2 21 in GABA $1.3 \pm 0.2 \mathrm{nC}, n=21 ; p=0.28$. PPR: control 1.4 $\pm 0.1, n=32$; Kir2.1 in glu $1.4 \pm 0.1, n=42$; $p=$ 0.77 ; control $1.1 \pm 0.1, n=25$, Kir2.1 in GABA $1.3 \pm 0.1, n=36$; $p=0.08)$.

Furthermore, in glutamatergic Kir2.1-expressing glu-GABA pairs, the mEPSCs recorded in GABAergic neurons had strongly reduced amplitude compared with the equivalent connection in control glu-GABA pairs, whereas the autaptic mEPSC were not affected by Kir2.1 expression (Fig. $9 A, B$ ). In addition, the difference in decay time of mEPSCs on GABAergic neurons compared with mEPSCs on glutamatergic neurons was abolished in glutamatergic Kir2.1-expressing glu-GABA pairs (decay time constant: control glu autapse: $3.5 \pm 0.1 \mathrm{~ms}, n=16$; control glu heterosynapse: $2.7 \pm 0.2 \mathrm{~ms}, n=15 ; p=0.0004$; Kir2.1 glu autapse: $3.4 \pm 0.1 \mathrm{~ms}, n=15$; Kir2.1 glu heterosynapse: $3.1 \pm$ $0.3 \mathrm{~ms}, n=14 ; p=0.31$ ). However, in GABAergic Kir2.1expressing glu-GABA pairs, the mEPSCs onto GABAergic or glutamatergic neurons were comparable to their respective group in control glu-GABA pairs (Fig. 9C,D). Therefore, these data indicate that in glu-GABA pairs, the output (e.g., RRP size and vesicle release efficiency) from and the synaptic input onto the GABAergic neuron are shaped by activity of the glutamatergic neuron.

\section{Discussion}

In this study, we used a simple two-neuron microcircuit to investigate the process of synaptic formation and evolvement of functional synaptic properties. We show that whereas glutamatergic neurons remained insensitive to glutamatergic or GABAergic innervation, GABAergic neurons exhibited modulation of both synaptic input and output properties in response to glutamatergic innervation. Although the findings are consistent with observations related to GABAergic synapse maturation (Marty et al., 2000; Morales et al., 2002; Chattopadhyaya et al., 2004; Colin-Le Brun et al., 2004; Tang et al., 2007; Jiang et al., 2010; Grantyn et al., 2011), we have now shown directly that these changes are due to glutamatergic input. GABAergic input modulation required firing in glutamatergic neurons, which led to a switch of postsynaptic AMPAR composition from linear to inward rectifying. GABAergic output changed through synapse proliferation accompanied by reduced release efficacy. Both firing in glutamatergic neurons and glutamate receptor activation were required to induce output modulation. The changes initiated in the GABAergic neuron were cell wide and not target-cell specific with regard to neurotransmitter identity, possibly suggesting the activation of a different genetic program with excitatory input.

\section{Mechanism of GABAergic output modulation}

The emergence of glutamatergic input onto GABAergic neurons increased the number of GABAergic synapses, but at the same time decreased their efficiency of release. Interestingly, both synapse proliferation and decreased release probability have been reported separately as hallmarks of GABAergic neuron maturation in the developing cortex (Morales et al., 2002; Chattopadhyaya et al., 2004; Tang et al., 2007; Jiang et al., 2010). The
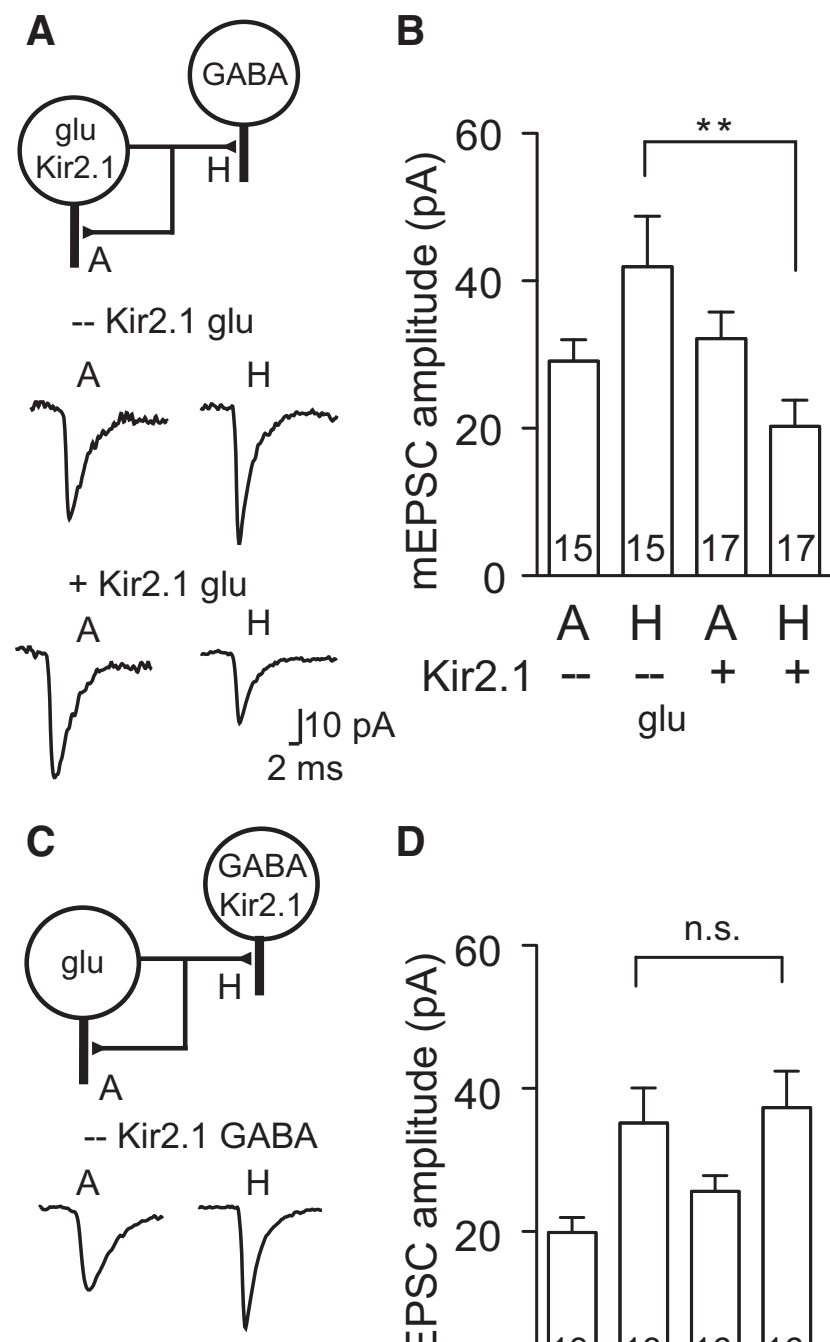

+ Kir2.1 GABA
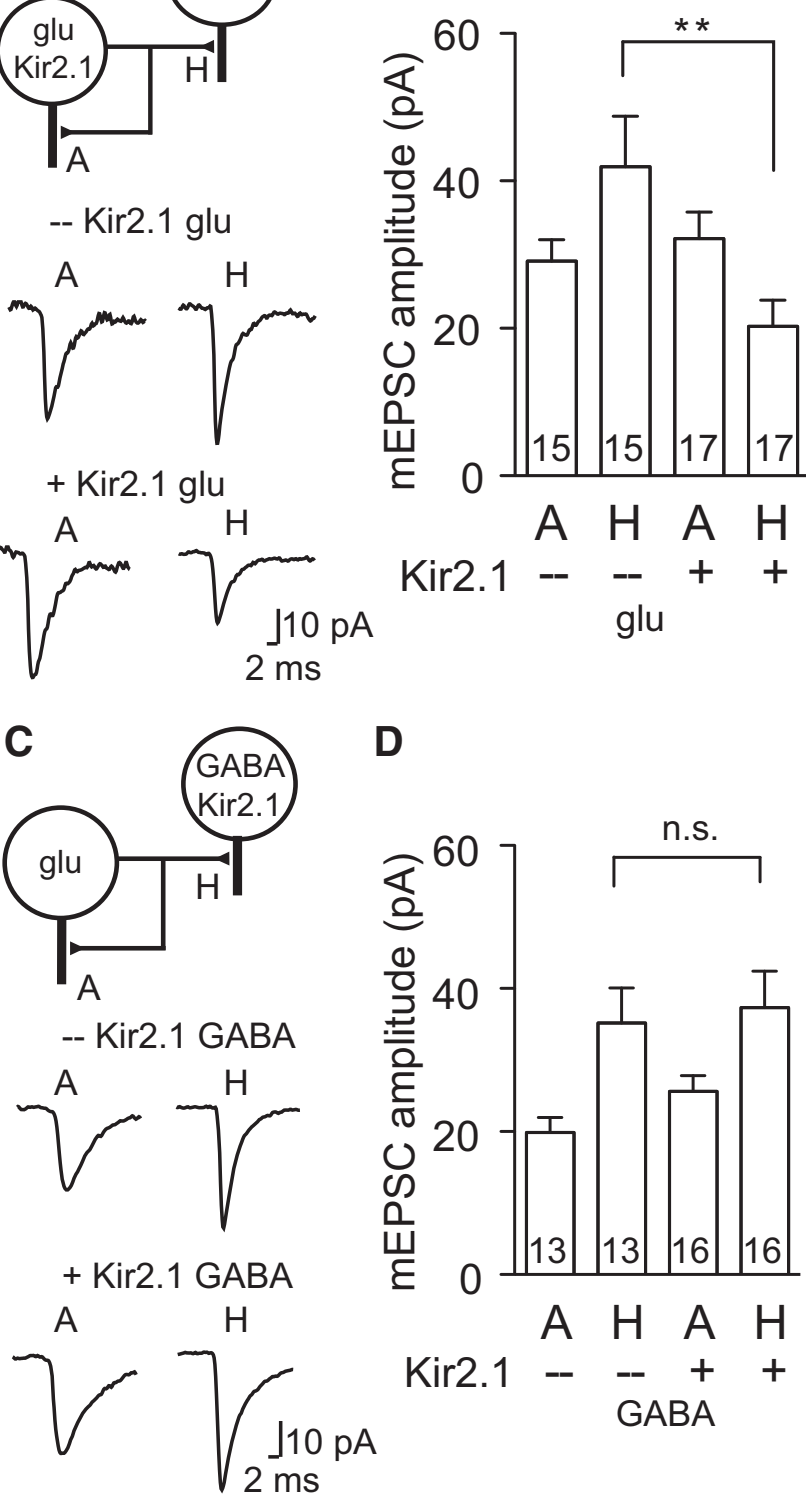

D

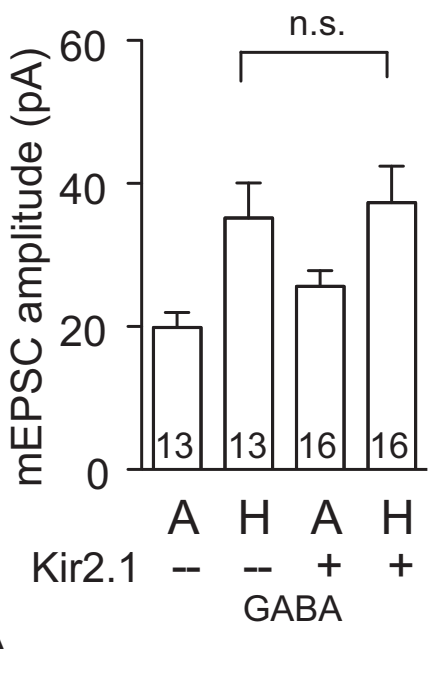

Figure 9. Suppression of glutamatergic neuronal activity by Kir2.1 prevents modulation of glutamatergic input onto GABAergic neurons in glu-GABA pairs. $\boldsymbol{A}$, Top, Schematic representation of a glu-GABA neuronal pair where Kir2.1 specifically expressed in a glutamatergic neuron. Bottom, Representative traces of mEPSCs recorded from glutamatergic (A: glutamatergic autapse) and GABAergic (H: glutamatergic heterosynapse) neurons in glu-GABA pairs where the glutamatergic cell is Kir2.1-expressing (+) or control vector infected (-). B, Bar graph showing the mean values of mEPSC amplitudes measured in glutamatergic $(A)$ or GABAergic $(H)$ neurons in glu-GABA pairs with a Kir2.1 expressing $(+)$ or control vector infected $(-)$ glutamatergic cell. ${ }^{* *} p \leq 0.01$. C, $\boldsymbol{D}$, Same as in $\boldsymbol{A}$ and $\boldsymbol{B}$, but with Kir2.1 expression in the GABAergic neuron in a glu-GABA pair. All values are mean \pm SEM. Numbers in bar graphs are $n$ values.

modulation that we observed required AP firing and the activation of ionotropic glutamate receptors (Fig. 6). Chronic blockade of $\mathrm{Ca}^{2+}$-permeable AMPARs also impaired the GABAergic output modification (data not shown), although it is unclear whether this was due to impaired $\mathrm{Ca}^{2+}$ influx into the GABAergic neuron or to reduced overall excitatory input. As for neuronal activity, expression of Kir2.1 in glutamatergic neurons alone reversed the GABAergic output properties, whereas expression of Kir2.1 in the GABAergic neuron did not. However, in the latter 
configuration, we still cannot rule out the possibility that the large input from the partner glutamatergic neuron in the microcircuit evokes firing in the GABAergic Kir2.1 neuron. Consistent with previous findings, we found that inactivity of glutamatergic neurons did not affect glutamatergic synapse formation (Craig et al., 1994; Rao and Craig, 1997; Gomperts et al., 2000; Lu et al., 2013).

What is the mechanism for the reduced $P_{\mathrm{vr}}$ accompanying the increased GABAergic synapse number? One possible explanation is that although making additional synapses requires the upregulation of hundreds of genes, gene products affecting synaptic output, such as voltage-gated channels, may not be upregulated proportionately, leading to their dilution over more synapses. Alternatively, active mechanisms could explain this result. For example, presynaptic release probability changes attributed to active $\mathrm{Ca}^{2+}$ modulation are observed when postsynaptic excitability is altered in Drosophila neuromuscular junction (Paradis et al., 2001) or global network activity is blocked in culture (Zhao et al., 2011).

\section{Activity-dependent modulation of AMPAR responses in GABAergic neurons}

Glutamatergic input is required for clustering AMPARs on hippocampal GABAergic neurons (Rao et al., 2000). We found that glutamatergic input also regulates the functional properties of these AMPARs. Glutamatergic responses in GABAergic neurons of the two-neuron microcircuit show inward rectification, large mEPSC amplitudes, and faster decay kinetics (Fig. 7), which is reminiscent of the GluA2-lacking AMPAR composition often observed in GABAergic neurons (McBain and Dingledine, 1993; Geiger et al., 1995; Isa et al., 1996; Racca et al., 1996). However, when AP firing in glutamatergic neurons was impeded, the AMPAR responses in GABAergic neurons showed characteristics of GluA2-containing AMPARs (Fig. 9). We suggest that the default AMPAR composition of synapses on GABAergic neurons is GluA2 containing and incorporation of GluA2-lacking AMPARs depends on glutamatergic neuronal activity and synaptic contact. The transition from $\mathrm{Ca}^{2+}$-impermeable to $\mathrm{Ca}^{2+}$-permeable AMPARs on GABAergic neurons after glutamatergic innervation may mark a step in the maturation of the GABAergic neuron. This is in contrast to more dynamic, activity-dependent modification of AMPAR composition, such as at the parallel fiber to stellate cell synapse in cerebellum, where high-frequency stimulation converts rectifying receptors to linearly conducting receptors (Liu and Cull-Candy, 2000; Liu and Cull-Candy, 2002).

Interestingly, ionotropic glutamate receptor antagonists did not prevent the incorporation of GluA2-lacking receptors into synapses on the GABAergic neurons. Therefore, initial synaptic contact, together with AP firing in the glutamatergic neuron, is sufficient for incorporation of inward-rectifying receptors. This result suggests that activity-dependent signals from glutamatergic neurons, such as AP-dependent release of trophic factors, might induce the expression of GluA2-lacking AMPARs on the GABAergic neuron, as has been suggested to occur in glutamatergic neurons (Nakata and Nakamura, 2007; Fortin et al., 2012).

\section{Cell intrinsic regulation in two-neuron culture system}

The properties of in vivo circuits are the result of intrinsic cellular programs and extrinsic network effects. In our reduced circuit, we suggest that glutamatergic neurons behave in a cell intrinsic fashion: they showed no change in synapse formation or function when cells received inputs from either glutamatergic or GABAergic neurons or experienced changes in activity levels. As further evidence of cell intrinsic behavior, we found that the total num- ber of synapses a glutamatergic neuron makes is not different between single and paired neurons. These results suggest that the glutamatergic neuron may be programmed to form a fixed number of connections, which is consistent with previous findings for glutamatergic neurons in microcircuits (Mennerick et al., 1995), in mass culture (Harms et al., 2005), and in vivo (Lu et al., 2013). Impeding AP firing in a postsynaptic neuron was shown to result in decreased glutamatergic synaptic contacts onto that neuron with respect to other neurons in the network (Burrone et al., 2002). However, global activity blockade does not result in decreased glutamatergic synapse formation (Craig et al., 1994; Rao and Craig, 1997; Gomperts et al., 2000; Burrone et al., 2002; Wierenga et al., 2006), suggesting that the effect of a postsynaptic neuron's activity on synaptic density is network dependent.

In the traditional sense, the changes induced in GABAergic neurons in response to glutamatergic innervation observed here are not cell intrinsic. It has been shown that silencing neuronal activity inhibits the formation of GABA synapses in a networkdependent fashion (Marty et al., 2000; Hartman et al., 2006). Consistent with these results, we found that the modulation of GABAergic input and output required the activity of a glutamatergic neuron (Figs. 8, 9). However, we interpret these changes as a maturational switch between cellular programs by glutamatergic input, because the changes were cell wide and not target-cell specific. GABAergic synapses contacting both postsynaptic cell types exhibited the same density and degree of decrease in release efficiency (Figs. 2, 5). In more complex networks, extrinsic factors likely further influence these processes, because synapse formation (Gulyás et al., 1990; Han et al., 1993; Larimer and Strowbridge, 2008) and short-term plasticity (Shigemoto et al., 1996; Thomson, 1997; Reyes et al., 1998; Scanziani et al., 1998; Gupta et al., 2000; Tóth and McBain, 2000; Fioravante and Regehr, 2011) exhibit target cell specificity.

GABAergic interneurons in hippocampus in vivo exhibit great morphological and functional heterogeneity (McBain and Fisahn, 2001; Somogyi and Klausberger, 2005). Although it has been shown that hippocampal GABAergic neurons in autaptic culture maintain functional diversity (Ikeda et al., 2008), we did not classify cells beyond neurotransmitter identity. Because glutamatergic input induced changes in GABAergic neurons, presumably over a range of subtypes, in our system, this may be a basic mechanism to induce maturation in the general population of inhibitory neurons.

We did not observe classical homeostatic plasticity changes such as scaling of synaptic strength (Turrigiano et al., 1998) with activity blockade in our two-neuron culture. However, it has been suggested that homeostatic scaling induced by activity blockade during synapse formation, a time range consistent with our TTX application, is a result of non-cell-autonomous network effects (Burrone et al., 2002; Hartman et al., 2006). We suggest our lack of synaptic scaling serves as further evidence that the two-neuron microcircuit is free of extrinsic network effects.

\section{A model for network development}

The modifications of GABAergic neuron input and output we observed in response to glutamatergic innervation would have several implications for the in vivo network. The coincident increase in GABAergic synapse formation and decrease in GABAergic synaptic efficiency initiated by glutamatergic innervation will change both the spatial and temporal properties of inhibition in a network. Because maturation of GABAergic synapses is an integral process to network development (Grantyn et al., 2011), it is important to understand how this process is affected by synaptic 
input on a basic level. Because this process appears to be preserved in the two-neuron microcircuit, we can use this model to further investigate the molecular pathways governing GABAergic maturation during normal development and how they may be perturbed in disease states.

\section{References}

Albert H, Dale EC, Lee E, Ow DW (1995) Site-specific integration of DNA into wild-type and mutant lox sites placed in the plant genome. Plant J 7:649-659. CrossRef Medline

Araki K, Araki M, Yamamura K (1997) Targeted integration of DNA using mutant lox sites in embryonic stem cells. Nucleic Acids Res 25:868-872. CrossRef Medline

Bats C, Soto D, Studniarczyk D, Farrant M, Cull-Candy SG (2012) Channel properties reveal differential expression of TARPed and TARPless AMPARs in stargazer neurons. Nat Neurosci 15:853-861. CrossRef Medline

Burrone J, O’Byrne M, Murthy VN (2002) Multiple forms of synaptic plasticity triggered by selective suppression of activity in individual neurons. Nature 420:414-418. CrossRef Medline

Chao HT, Chen H, Samaco RC, Xue M, Chahrour M, Yoo J, Neul JL, Gong S, Lu HC, Heintz N, Ekker M, Rubenstein JL, Noebels JL, Rosenmund C, Zoghbi HY (2010) Dysfunction in GABA signalling mediates autismlike stereotypies and Rett syndrome phenotypes. Nature 468:263-269. CrossRef Medline

Chattopadhyaya B, Di Cristo G, Higashiyama H, Knott GW, Kuhlman SJ, Welker E, Huang ZJ (2004) Experience and activity-dependent maturation of perisomatic GABAergic innervation in primary visual cortex during a postnatal critical period. J Neurosci 24:9598-9611. CrossRef Medline

Clements JD, Bekkers JM (1997) Detection of spontaneous synaptic events with an optimally scaled template. Biophys J 73:220-229. CrossRef Medline

Cohen D, Segal M (2011) Network bursts in hippocampal microcultures are terminated by exhaustion of vesicle pools. J Neurophysiol 106:23142321. CrossRef Medline

Colin-Le Brun I, Ferrand N, Caillard O, Tosetti P, Ben-Ari Y, Gaïarsa JL (2004) Spontaneous synaptic activity is required for the formation of functional GABAergic synapses in the developing rat hippocampus. J Physiol 559:129-139. CrossRef Medline

Craig AM, Boudin H (2001) Molecular heterogeneity of central synapses: afferent and target regulation. Nat Neurosci 4:569-578. CrossRef Medline

Craig AM, Blackstone CD, Huganir RL, Banker G (1994) Selective clustering of glutamate and gamma-aminobutyric acid receptors opposite terminals releasing the corresponding neurotransmitters. Proc Natl Acad Sci U S A 91:12373-12377. CrossRef Medline

Fioravante D, Regehr WG (2011) Short-term forms of presynaptic plasticity. Curr Opin Neurobiol 21:269-274. CrossRef Medline

Flavell SW, Greenberg ME (2008) Signaling mechanisms linking neuronal activity to gene expression and plasticity of the nervous system. Annu Rev Neurosci 31:563-590. CrossRef Medline

Fortin DA, Srivastava T, Dwarakanath D, Pierre P, Nygaard S, Derkach VA, Soderling TR (2012) Brain-derived neurotrophic factor activation of CaM-kinase kinase via transient receptor potential canonical channels induces the translation and synaptic incorporation of GluA1-containing calcium-permeable AMPA receptors. J Neurosci 32:8127-8137. CrossRef Medline

Geiger JR, Melcher T, Koh DS, Sakmann B, Seeburg PH, Jonas P, Monyer H (1995) Relative abundance of subunit mRNAs determines gating and $\mathrm{Ca}^{2+}$ permeability of AMPA receptors in principal neurons and interneurons in rat CNS. Neuron 15:193-204. CrossRef Medline

Geiger JR, Lübke J, Roth A, Frotscher M, Jonas P (1997) Submillisecond AMPA receptor-mediated signaling at a principal neuron-interneuron synapse. Neuron 18:1009-1023. CrossRef Medline

Ghosh A, Ginty DD, Bading H, Greenberg ME (1994) Calcium regulation of gene expression in neuronal cells. J Neurobiol 25:294-303. CrossRef Medline

Gomperts SN, Carroll R, Malenka RC, Nicoll RA (2000) Distinct roles for ionotropic and metabotropic glutamate receptors in the maturation of excitatory synapses. J Neurosci 20:2229-2237. Medline

Grantyn R, Henneberger C, Jüttner R, Meier JC, Kirischuk S (2011) Func- tional hallmarks of GABAergic synapse maturation and the diverse roles of neurotrophins. Front Cell Neurosci 5:13. Medline

Gulyás AI, Gorcs TJ, Freund TF (1990) Innervation of different peptidecontaining neurons in the hippocampus by GABAergic septal afferents. Neuroscience 37:31-44. CrossRef Medline

Gupta A, Wang Y, Markram H (2000) Organizing principles for a diversity of GABAergic interneurons and synapses in the neocortex. Science 287: 273-278. CrossRef Medline

Han ZS, Buhl EH, Lörinczi Z, Somogyi P (1993) A high degree of spatial selectivity in the axonal and dendritic domains of physiologically identified local-circuit neurons in the dentate gyrus of the rat hippocampus. Eur J Neurosci 5:395-410. CrossRef Medline

Harms KJ, Tovar KR, Craig AM (2005) Synapse-specific regulation of AMPA receptor subunit composition by activity. J Neurosci 25:63796388. CrossRef Medline

Hartman KN, Pal SK, Burrone J, Murthy VN (2006) Activity-dependent regulation of inhibitory synaptic transmission in hippocampal neurons. Nat Neurosci 9:642-649. CrossRef Medline

Huang ZJ (2009) Activity-dependent development of inhibitory synapses and innervation pattern: role of GABA signalling and beyond. J Physiol 587:1881-1888. CrossRef Medline

Ikeda K, Yanagawa Y, Bekkers JM (2008) Distinctive quantal properties of neurotransmission at excitatory and inhibitory autapses revealed using variance-mean analysis. J Neurosci 28:13563-13573. CrossRef Medline

Isaac JT, Ashby MC, McBain CJ (2007) The role of the GluR2 subunit in AMPA receptor function and synaptic plasticity. Neuron 54:859-871. CrossRef Medline

Isa T, Itazawa S, Iino M, Tsuzuki K, Ozawa S (1996) Distribution of neurones expressing inwardly rectifying and $\mathrm{Ca}(2+)$-permeable AMPA receptors in rat hippocampal slices. J Physiol 491:719-733. Medline

Jiang B, Huang S, de Pasquale R, Millman D, Song L, Lee HK, Tsumoto T, Kirkwood A (2010) The maturation of GABAergic transmission in visual cortex requires endocannabinoid-mediated LTD of inhibitory inputs during a critical period. Neuron 66:248-259. CrossRef Medline

Jonas P, Burnashev N (1995) Molecular mechanisms controlling calcium entry through AMPA-type glutamate receptor channels. Neuron 15:987990. CrossRef Medline

Kim JH, Lee SR, Li LH, Park HJ, Park JH, Lee KY, Kim MK, Shin BA, Choi SY (2011) High cleavage efficiency of a 2A peptide derived from porcine teschovirus-1 in human cell lines, zebrafish and mice. PLoS One 6:e18556. CrossRef Medline

Klausberger T, Somogyi P (2008) Neuronal diversity and temporal dynamics: the unity of hippocampal circuit operations. Science 321:53-57. CrossRef Medline

Knight ZA, Tan K, Birsoy K, Schmidt S, Garrison JL, Wysocki RW, Emiliano A, Ekstrand MI, Friedman JM (2012) Molecular profiling of activated neurons by phosphorylated ribosome capture. Cell 151:1126-1137. CrossRef Medline

Larimer P, Strowbridge BW (2008) Nonrandom local circuits in the dentate gyrus. J Neurosci 28:12212-12223. CrossRef Medline

Liu SJ, Cull-Candy SG (2002) Activity-dependent change in AMPA receptor properties in cerebellar stellate cells. J Neurosci 22:3881-3889. Medline

Liu SQ, Cull-Candy SG (2000) Synaptic activity at calcium-permeable AMPA receptors induces a switch in receptor subtype. Nature 405:454458. CrossRef Medline

Lois C, Hong EJ, Pease S, Brown EJ, Baltimore D (2002) Germline transmission and tissue-specific expression of transgenes delivered by lentiviral vectors. Science 295:868-872. CrossRef Medline

Lu B, Wang KH, Nose A (2009) Molecular mechanisms underlying neural circuit formation. Curr Opin Neurobiol 19:162-167. CrossRef Medline

Lu W, Bushong EA, Shih TP, Ellisman MH, Nicoll RA (2013) The cellautonomous role of excitatory synaptic transmission in the regulation of neuronal structure and function. Neuron 78:433-439. CrossRef Medline

Marty S, Wehrlé R, Sotelo C (2000) Neuronal activity and brain-derived neurotrophic factor regulate the density of inhibitory synapses in organotypic slice cultures of postnatal hippocampus. J Neurosci 20:8087-8095. Medline

McBain CJ, Dingledine R (1993) Heterogeneity of synaptic glutamate receptors on CA3 stratum radiatum interneurones of rat hippocampus. J Physiol 462:373-392. Medline 
McBain CJ, Fisahn A (2001) Interneurons unbound. Nat Rev Neurosci 2:11-23. CrossRef Medline

Mennerick S, Que J, Benz A, Zorumski CF (1995) Passive and synaptic properties of hippocampal neurons grown in microcultures and in mass cultures. J Neurophysiol 73:320-332. Medline

Meyuhas O (2008) Physiological roles of ribosomal protein S6: one of its kind. Int Rev Cell Mol Biol 268:1-37. CrossRef Medline

Morales B, Choi SY, Kirkwood A (2002) Dark rearing alters the development of GABAergic transmission in visual cortex. J Neurosci 22:80848090. Medline

Morgan JI, Curran T (1991) Stimulus-transcription coupling in the nervous system: involvement of the inducible proto-oncogenes fos and jun. Annu Rev Neurosci 14:421-451. CrossRef Medline

Nakata H, Nakamura S (2007) Brain-derived neurotrophic factor regulates AMPA receptor trafficking to post-synaptic densities via IP3R and TRPC calcium signaling. FEBS Lett 581:2047-2054. CrossRef Medline

Paradis S, Sweeney ST, Davis GW (2001) Homeostatic control of presynaptic release is triggered by postsynaptic membrane depolarization. Neuron 30:737-749. CrossRef Medline

Racca C, Catania MV, Monyer H, Sakmann B (1996) Expression of AMPAglutamate receptor B subunit in rat hippocampal GABAergic neurons. Eur J Neurosci 8:1580-1590. CrossRef Medline

Rao A, Craig AM (1997) Activity regulates the synaptic localization of the NMDA receptor in hippocampal neurons. Neuron 19:801-812. CrossRef Medline

Rao A, Cha EM, Craig AM (2000) Mismatched appositions of presynaptic and postsynaptic components in isolated hippocampal neurons. J Neurosci 20:8344-8353. Medline

Reyes A, Lujan R, Rozov A, Burnashev N, Somogyi P, Sakmann B (1998) Target-cell-specific facilitation and depression in neocortical circuits. Nat Neurosci 1:279-285. CrossRef Medline

Rosenmund C, Stevens CF (1996) Definition of the readily releasable pool of vesicles at hippocampal synapses. Neuron 16:1197-1207. CrossRef Medline

Rubenstein JL, Merzenich MM (2003) Model of autism: increased ratio of excitation/inhibition in key neural systems. Genes Brain Behav 2:255267. CrossRef Medline

Scanziani M, Gähwiler BH, Charpak S (1998) Target cell-specific modulation of transmitter release at terminals from a single axon. Proc Natl Acad Sci U S A 95:12004-12009. CrossRef Medline

Shigemoto R, Kulik A, Roberts JD, Ohishi H, Nusser Z, Kaneko T, Somogyi P (1996) Target-cell-specific concentration of a metabotropic glutamate receptor in the presynaptic active zone. Nature 381:523-525. CrossRef Medline

Sohal VS, Zhang F, Yizhar O, Deisseroth K (2009) Parvalbumin neurons and gamma rhythms enhance cortical circuit performance. Nature 459: 698-702. CrossRef Medline

Somogyi P, Klausberger T (2005) Defined types of cortical interneurone structure space and spike timing in the hippocampus. J Physiol 562:9-26. CrossRef Medline

Tamamaki N, Yanagawa Y, Tomioka R, Miyazaki J, Obata K, Kaneko T (2003) Green fluorescent protein expression and colocalization with calretinin, parvalbumin, and somatostatin in the GAD67-GFP knock-in mouse. J Comp Neurol 467:60-79. CrossRef Medline

Tang AH, Chai Z, Wang SQ (2007) Dark rearing alters the short-term synaptic plasticity in visual cortex. Neurosci Lett 422:49-53. CrossRef Medline

Thomson AM (1997) Activity-dependent properties of synaptic transmission at two classes of connections made by rat neocortical pyramidal axons in vitro. J Physiol 502:131-147. CrossRef Medline

Tóth K, McBain CJ (2000) Target-specific expression of pre- and postsynaptic mechanisms. J Physiol 525:41-51.

Turrigiano GG, Nelson SB (2004) Homeostatic plasticity in the developing nervous system. Nat Rev Neurosci 5:97-107. CrossRef Medline

Turrigiano GG, Leslie KR, Desai NS, Rutherford LC, Nelson SB (1998) Activity-dependent scaling of quantal amplitude in neocortical neurons. Nature 391:892-896. CrossRef Medline

Varoqueaux F, Sigler A, Rhee JS, Brose N, Enk C, Reim K, Rosenmund C (2002) Total arrest of spontaneous and evoked synaptic transmission but normal synaptogenesis in the absence of Munc13-mediated vesicle priming. Proc Natl Acad Sci U S A 99:9037-9042. CrossRef Medline

Verhage M, Maia AS, Plomp JJ, Brussaard AB, Heeroma JH, Vermeer H, Toonen RF, Hammer RE, van den Berg TK, Missler M, Geuze HJ, Südhof TC (2000) Synaptic assembly of the brain in the absence of neurotransmitter secretion. Science 287:864-869. CrossRef Medline

Wierenga CJ, Walsh MF, Turrigiano GG (2006) Temporal regulation of the expression locus of homeostatic plasticity. J Neurophysiol 96:2127-2133. CrossRef Medline

Williams ME, Wilke SA, Daggett A, Davis E, Otto S, Ravi D, Ripley B, Bushong EA, Ellisman MH, Klein G, Ghosh A (2011) Cadherin-9 regulates synapse-specific differentiation in the developing hippocampus. Neuron 71:640-655. CrossRef Medline

Xue M, Reim K, Chen X, Chao HT, Deng H, Rizo J, Brose N, Rosenmund C (2007) Distinct domains of complexin I differentially regulate neurotransmitter release. Nat Struct Mol Biol 14:949-958. CrossRef Medline

Zhao C, Dreosti E, Lagnado L (2011) Homeostatic synaptic plasticity through changes in presynaptic calcium influx. J Neurosci 31:7492-7496. CrossRef Medline

Zhu Y, Xu J, Heinemann SF (2009) Two pathways of synaptic vesicle retrieval revealed by single-vesicle imaging. Neuron 61:397-411. CrossRef Medline 